\title{
Effect of spherical pores coalescence on the overall conductivity of a material.
}

\author{
L. Lanzoni ${ }^{1}$, E. Radi²,3 I. Sevostianov*4 \\ ${ }^{1}$ Dipartimento di Ingegneria "Enzo Ferrari”, Università di Modena e Reggio Emilia, Via Vivarelli, 10- 41125 Modena, \\ Italy. \\ ${ }^{2}$ Dipartimento di Scienze e Metodi dell'Ingegneria, Università di Modena e Reggio Emilia, Via Amendola, 2 - 42122 \\ Reggio Emilia, Italy. \\ ${ }^{3}$ Centro Interdipartimentale “En\&Tech”, via G. Amendola, 2, Reggio Emilia, 42122, Italy \\ ${ }^{4}$ Department of Mechanical and Aerospace Engineering, New Mexico State University, Las Cruces, NM 88001, USA. \\ * Author for correspondence: igor@nmsu.edu
}

\begin{abstract}
The problem about steady-state temperature distribution in a homogeneous isotropic medium containing a pore or an insulating inhomogeneity formed by two coalesced spheres of the same radius, under arbitrarily oriented uniform heat flux, is solved analytically. The limiting case of two touching spheres is analyzed separately. The solution is obtained in the form of converged integrals that can be calculated using Gauss-Laguerre quadrature rule. The temperature on the inhomogeneity's surface is used to determine components of the resistivity contribution tensor for the insulating inhomogeneity of the mentioned shape. An interesting observation is that the extreme values of these components are achieved when the spheres are already slightly coalesced.
\end{abstract}

Keywords: double-sphere pore, temperature field, conductivity, Legendre functions, toroidal coordinates, Laplace's equation.

\section{Introduction.}

The purpose of this work is to evaluate the effect of two coalesced spherical pores or insulating inhomogeneities on the overall conductive properties. It is well known that the pore coalescence is typical, for example in the processes of sintering (Olevsky, 1998), heat treatment of cold sprayed and thermally sprayed coatings (Zhou et al., 2019), accumulation of radiation damage (Porollo et al, 2006), etc. As shown by Sevostianov and Kachanov (2015), such microstructural changes affect both the 
overall properties and local fields in the materials. At the same time, analytical modeling of the properties of materials with microstructures formed by inhomogeneities of non-ellipsoidal shape has not been well developed. The inhomogeneities are typically assumed to be ellipsoids of identical aspect ratios. This unrealistic assumption is largely responsible for insufficient linkage between methods of micromechanics and materials science applications. The reason for this lack is quite obvious: while for 2-D non-elliptical inhomogeneities many analytical and numerical results have been obtained, only a limited number of numerical results and approximate estimates are available for non-ellipsoidal 3-D shapes (see discussion in the book of Kachanov and Sevostianov, 2018). Among exact analytical results for effects of non-ellipsoidal inhomogeneities, we can only mention those of Kachanov and Sevostianov (2012) (effect of cracks growing from pores and cracks with partial contact between the faces on the overall elastic compliance), Radi and Sevostianov (2016) (effect of a toroidal inhomogeneity on the effective conductive properties), Argatov and Sevostianov (2011) and Krasnitskii et al. (2019) (effects of thin or arbitrary, respectively, rigid tori on the overall elastic properties).

The main goal of this paper is to obtain an analytical solution for components of the resistivity contribution tensor of a pore or insulating particle formed by two overlapping spheres. In a certain sense, we extend the two-dimensional analytical solution for the resistivity contribution tensor of two coalesced circular pores provided by Lanzoni et al. (2018) to the three-dimensional case.

The resistivity contribution tensor gives the extra temperature gradient produced by introduction of the inhomogeneity into a material subjected to otherwise uniform heat flux. This tensor has been introduced by Sevostianov and Kachanov (2002). Following this work, we assume that the background material of volume $V$ having the isotropic thermal conductivity $k$ contains an isolated pore or insulating inhomogeneity of volume $V_{1}$. Assuming linear Fourier relation between the temperature gradient $\nabla T$ and the heat flux vector $\boldsymbol{q}$ per reference volume, the change in $\nabla T$, required to maintain the same heat flux if the inhomogeneity is introduced, is given by

$$
\Delta(\nabla T)=\frac{V_{1}}{V} \boldsymbol{R} \cdot \boldsymbol{q},
$$

where the symmetric second-rank tensor $\boldsymbol{R}$ is the resistivity contribution tensor of the inhomogeneity. The additional temperature gradient due to the presence of an insulating inhomogeneity can be represented as the following integral over the inhomogeneity boundary

$$
\Delta(\nabla T)=\frac{1}{V} \int_{S} T \boldsymbol{n} d S
$$


where $T$ and $\boldsymbol{n}$ are temperature and the outward unit vector, normal to the boundary $S$, respectively.

To the best of our knowledge the first solution of conductivity problem for two spheres has been obtained by Jeffery (1912) who used bi-spherical coordinates and produced a solution in the form of infinite series in Legendre polynomials. As shown by Chowdhury and Christov (2010), the convergence of this series is very fast (exponential). The general form of the solution of Laplace equation in bispherical coordinates has been given by Stoy (1986 a, b). Sevostianov et al (2014) proposed a simple method of estimating the effect of interaction of two spherical pores or inhomogeneities on the overall elastic and conductive properties. They approximately evaluated components of compliance and resistivity contribution tensors and compare their results with numerical calculations.

In the context of overlapping spheres, the problem of polarizability has been first addressed by Schiffer and Szego (1949) who obtained a solution in integral form. Later the problem was readdressed by Felderhof and Palaniappan (1999, 2000), who used Mehler-Fock transform to obtain an exact expression in integral form for the electrostatic capacitance of two overlapping conducting spheres of arbitrary radii with arbitrary angle of intersection. The integral can be evaluated if the angle of intersection can be expressed as $m \pi / n$ with $m$ and $n$ integers. The solution can be used to evaluate the steady-state heat flux from the hot body placed in a thermally conducting environment of lower temperature. Pitkonen $(2006,2007)$ considered dielectric overlapping spheres and obtained solutions in the form of an integral of a parameter given by a Neumann series. He also considered the problem about touching spheres (Pitkonen, 2008) and analyzed it as an eigenfunction expansion in the tangent sphere coordinate system. Liemert (2014) obtained an explicit solution of Laplace's equation for the two conducting overlapping spheres placed in a uniform longitudinal and transverse exciting electric field and assumed to be at constant potential. When spheres intersect at an angle $\pi / n$, the problem is solvable in elementary form via the method of images (Tong, 1996; Palaniappan, 2012), which may result effective also for the problem of insulating coalesced spheres considered here. Note, however, that the mentioned works focused at Dirichlet problem (i.e. scattered potential has been evaluated when incident potential is applied) that does not allow explicit evaluation of the resistivity contribution tensors, the primary focus of the present paper.

In the text to follow, we solve the Neumann boundary value problem for an infinite domain containing a pore (or insulating inhomogeneity) in the shape of two overlapping spheres that first touch each other then coalesce and finally gradually collapse to a penny shaped crack. We treat the cases of 
longitudinal and transverse heat flux separately. Due to the linear character of the problem, the case of arbitrary oriented flux is then recovered by superposition. Our primary goal is to find the temperature field at the pore surface. From these data, we calculate for the first time the components of resistivity contribution tensor using equations (1.1) and (1.2). We also show that the contribution of the two overlapped spherical pores to the overall conductivity can be approximated with good accuracy by that given by a spheroid of a well definite aspect ratio that we explicitly calculate. The limiting case of two spherical pores touching each other at one point is considered separately by using the tangent sphere coordinate system.

\section{Formulation of the problem in toroidal coordinates.}

We consider a pore (or a perfectly insulating inhomogeneity) embedded in an infinite conductive medium subjected to a remotely applied uniform heat flux. The boundary condition at the pore surface is zero normal component of the heat flux. Following Morse and Feshbach (1953) and Lebedev et al (1965), we introduce toroidal coordinate system $(\alpha, \beta, \gamma)$ defined by the following relations

$$
x=\frac{a \sinh \alpha \cos \gamma}{\cosh \alpha-\cos \beta}, \quad y=\frac{a \sinh \alpha \sin \gamma}{\cosh \alpha-\cos \beta}, \quad z=\frac{a \sin \beta}{\cosh \alpha-\cos \beta} .
$$

where $a$ denotes the radius of the circle resulted from the intersection of spheres, $\alpha \in(-\infty, \infty), \beta \in$ $[-\pi, \pi]$ and $\gamma \in[0,2 \pi]$ (Figure 1).

The constant values $\pm \beta_{0}$ of the coordinate $\beta$ define a double-sphere surface of radius $R$, with sphere centers laying on $z$-axis at $z=z_{0}$ (see Fig. 1a) :

$$
R=a / \sin \beta_{0}, \quad z_{0}= \pm R \cos \beta_{0} .
$$

Variation of $\beta_{0}$ from 0 to $\pi / 2$ corresponds to the variation of the shape of two coalesced spherical pores: from point contact to partial or complete coalescence. Further increase of $\beta_{0}$ corresponds to gradual collapse of the sphere to a lentil and further to a penny-shaped crack (Figure 2).

The temperature distribution $T$ under steady-state heat flux satisfies the Laplace equation

$$
\nabla^{2} T=0 .
$$

The heat flux is given by the isotropic Fourier law

$$
\boldsymbol{q}=-k \nabla T,
$$

where $k$ is the heat conduction coefficient. Then, the boundary condition on the pore surface, requires

$$
\boldsymbol{q} \cdot \boldsymbol{n}=0, \quad \text { at } \beta= \pm \beta_{0}
$$


where $\boldsymbol{n}$ is the outer unit normal on the double-sphere surface (Fig.1), coinciding with the unit vector $-\boldsymbol{e}_{\beta}$. Thus, the temperature field must satisfy the Neumann boundary condition on the pore surface:

$$
\frac{\partial T}{\partial \beta}=0 \quad \text { at } \beta= \pm \beta_{0}
$$

At large distance from the pore (both $\alpha$ and $\beta$ tend to zero) the heat flux must approach the remotely applied one $\boldsymbol{q}=\boldsymbol{q}_{0}$. Below we consider two cases when $\boldsymbol{q}_{0}$ directed along the symmetry axis of the pore and transverse to it.

\section{Heat flux along the symmetry axis.}

We first consider the axisymmetric problem corresponding to a remote heat flux $\boldsymbol{q}_{0}=\left(0,0, q_{0}\right)$ along the symmetry axis of the pore. Following Radi and Sevostianov (2016), we split the temperature field into the sum $T=T_{0}+T_{1}$ where $T_{0}$ is the fundamental contribution induced by the uniform heat flux in a homogeneous medium and $T_{1}$ denotes the perturbation temperature field due to the presence of the coalesced spherical cavities. According to $(2.1)_{3}$, the fundamental contribution is given by

$$
T_{0}(\alpha, \beta)=-\frac{q_{0}}{k} z=-\frac{q_{0} R}{k} \frac{\sin \beta_{0} \sin \beta}{\cosh \alpha-\cos \beta}
$$

(with the accuracy of arbitrary additive constant defining the reference temperature). The field $T_{0}$ is harmonic and skew-symmetric with respect to $\beta$. Then, equation (2.3) implies that the field $T_{1}$ must also be harmonic and skew-symmetric. Without losing the generality, we can consider only the upper half-space, where $\beta$ is positive. The most general harmonic axisymmetric function in toroidal coordinates, which is odd in $\beta$, has the following form (Morse and Feshbach, 1953; Sneddon, 1972)

$$
T_{1}(\alpha, \beta)=\frac{q_{0} R}{k} \sin \beta_{0} \sqrt{\cosh \alpha-\cos \beta} \int_{0}^{\infty} u(\tau) P_{-1 / 2+i \tau}(\cosh \alpha) \sinh \tau \beta \mathrm{d} \tau,
$$

where $P_{-1 / 2+i \tau}$ is the Legendre function of the first kind with complex index (Bateman and Erdelyi, 1953; Lebedev et al., 1965), which is vanishing at infinity. The unknown function $u(\tau)$ must be found by imposing the boundary condition (2.6) at $\beta=\beta_{0}$ (where $\beta_{0}>0$ ):

$$
\frac{\sin \beta_{0}}{2\left(\cosh \alpha-\cos \beta_{0}\right)} \int_{0}^{\infty} u(\tau) P_{-1 / 2+i \tau}(\cosh \alpha) \sinh \tau \beta_{0} \mathrm{~d} \tau+\int_{0}^{\infty} \tau u(\tau) P_{-1 / 2+i \tau}(\cosh \alpha) \cosh \tau \beta_{0} \mathrm{~d} \tau=
$$




$$
=\frac{\cosh \alpha \cos \beta_{0}-1}{\left(\cosh \alpha-\cos \beta_{0}\right)^{5 / 2}} .
$$

Then, using the orthogonality relation (Gradshteyn and Ryzhik, 2007)

$$
\int_{0}^{\infty} P_{-1 / 2+i \tau}(\cosh \alpha) P_{-1 / 2+i \eta}(\cosh \alpha) \sinh \alpha \mathrm{d} \alpha=\frac{\delta(\tau-\eta)}{\tau \tanh \tau \pi}
$$

and expression (A.5) derived in the Appendix, one gets the following Fredholm integral equation of the second kind for the unknown function $u(\tau)$

$$
u(\tau) \frac{\cosh \tau \beta_{0}}{\tanh \tau \pi}+\frac{\sin \beta_{0}}{2} \int_{0}^{\infty} U(\tau, \eta) u(\eta) \sinh \eta \beta_{0} \mathrm{~d} \eta=\frac{2 \sqrt{2}}{3} \frac{\cot \beta_{0} \sinh \tau\left(\pi-\beta_{0}\right)-2 \tau \cosh \tau\left(\pi-\beta_{0}\right)}{\sinh \tau \pi}
$$

with the subkernel

$$
U(\tau, \eta)=\int_{0}^{\infty} \frac{P_{-1 / 2+i \tau}(\cosh \alpha) P_{-1 / 2+i \eta}(\cosh \alpha)}{\cosh \alpha-\cos \beta_{0}} \sinh \alpha \mathrm{d} \alpha=\int_{1}^{\infty} \frac{P_{-1 / 2+i \tau}(s) P_{-1 / 2+i \eta}(s)}{s-\cos \beta_{0}} \mathrm{~d} s,
$$

Note that $U(\tau, \eta)=U(\eta, \tau)$ (Pitkonen, 2007) and integral in (3.6) converges since $P_{-1 / 2+i \tau}(\cosh \alpha)$ decays as $e^{-\alpha / 2}$ as $\alpha$ tends to infinity. The numerical calculation of the subkernel $U(\tau, \eta)$ is addressed in Appendix B by exploiting the asymptotic behavior of the Legendre functions as $s \rightarrow \infty$.

The substitution suggested by Cochran (1972),

$$
v(\tau)=u(\tau) \sqrt{\frac{\sinh \tau \beta_{0} \cosh \tau \beta_{0}}{\tanh \tau \pi}},
$$

then transforms equation (3.5) into a Fredholm integral equation for the function $v(\tau)$ in the usual form

$$
v(\tau)+\frac{\sin \beta_{0}}{2} \int_{0}^{\infty} K(\tau, \eta) v(\eta) d \eta=f(\tau)
$$

where

$$
f(\tau)=\frac{4}{3} \sqrt{\frac{\tanh \tau \beta_{0}}{\sinh 2 \tau \pi}}\left[\cot \beta_{0} \sinh \tau\left(\pi-\beta_{0}\right)-2 \tau \cosh \tau\left(\pi-\beta_{0}\right)\right],
$$

and

$$
K(\tau, \eta)=\sqrt{\tanh \tau \pi \tanh \tau \beta_{0}} U(\tau, \eta) \sqrt{\tanh \eta \pi \tanh \eta \beta_{0}},
$$

is the symmetric kernel. 


\subsection{Numerical solution of the Fredholm integral equation}

The numerical solution to the Fredholm integral equation (3.9) can be obtained using the Nyström method. We apply the Gauss-Laguerre quadrature rule for the semi-infinite interval to equation (3.9), obtaining

$$
v(\tau)=-\frac{\sin \beta_{0}}{2} \sum_{j=1}^{n} w_{j} K\left(\tau, \eta_{j}\right) v\left(\eta_{j}\right)+f(\tau),
$$

where $\eta_{j}(j=1,2, \ldots, n)$ are the Gauss-Laguerre quadrature points, coinciding with the roots of the Laguerre polynomial $L_{n}(\eta)$ of degree $n$, and

$$
w_{j}=\frac{\eta_{j} e^{\eta_{j}}}{(n+1)^{2}\left[L_{n+1}\left(\eta_{j}\right)\right]^{2}}, \quad(j=1,2, \ldots, n)
$$

are the corresponding weights (Abramowitz and Stegun, 1972). Eqn (3.12) evaluated at the quadrature points then yields

$$
v\left(\tau_{i}\right)=-\frac{\sin \beta_{0}}{2} \sum_{j=1}^{n} w_{j} K\left(\tau_{i}, \eta_{j}\right) v\left(\eta_{j}\right)+f\left(\tau_{i}\right) .
$$

Let $v_{i}=v\left(\tau_{i}\right), f_{i}=f\left(\tau_{i}\right)$, and $K_{i j}=w_{j} K\left(\tau_{i}, \eta_{j}\right)$, then in matrix notation eqn (3.14) becomes

$$
\left(\mathbf{I}+\frac{\sin \beta_{0}}{2} \mathbf{K}\right) \mathbf{v}=\mathbf{f},
$$

where $\mathbf{I}$ is the identity matrix. This is a set of $n$ linear algebraic equations in $n$ unknowns that can be solved for $\mathbf{v}$ by standard techniques. Note that the matrix $\mathbf{K}$ is not symmetric, due to the weights $w_{j}$. The solution of (3.15) is expected to be well-conditioned everywhere except of $\beta_{0}=0$ since the matrix operator in (3.15) is bijective and non-singular. It follows from the properties of subkernel $U(\tau, \eta)$ and formulas (3.11) and (3.13) for kernel $K(\tau, \eta)$ and weight functions $w_{j}$. Case $\beta_{0}=0$ is considered separately in Section 6.

\subsection{Temperature field}

Once the unknowns $v_{j}$, for $j=1,2, \ldots, n$, have been obtained from the solution of the linear system (3.15), the perturbation temperature field follows from (3.2) and (3.8) as 


$$
T_{1}(\alpha, \beta)=\frac{q_{0} R}{k} \sin \beta_{0} \sqrt{\cosh \alpha-\cos \beta} \int_{0}^{\infty} v(\tau) \sqrt{\frac{\tanh \tau \pi}{\sinh \tau \beta_{0} \cosh \tau \beta_{0}}} P_{-1 / 2+i \tau}(\cosh \alpha) \sinh \tau \beta \mathrm{d} \tau,(3
$$

and, thus, the perturbation temperature field on the cavity surface at $\beta=\beta_{0}$ is given by

$$
T_{1}\left(\alpha, \beta_{0}\right)=\frac{q_{0} R}{k} \sin \beta_{0} \sqrt{\cosh \alpha-\cos \beta_{0}} \int_{0}^{\infty} v(\tau) \sqrt{\tanh \tau \pi \tanh \tau \beta_{0}} P_{-1 / 2+i \tau}(\cosh \alpha) \mathrm{d} \tau .
$$

The latter integral can be calculated by Gauss-Laguerre quadrature rule, namely

$$
T_{1}\left(\alpha, \beta_{0}\right)=\frac{q_{0} R}{k} \sin \beta_{0} \sqrt{\cosh \alpha-\cos \beta_{0}} \sum_{j=1}^{n} w_{j} v_{j} \sqrt{\tanh \eta_{j} \pi \tanh \eta_{j} \beta_{0}} P_{-1 / 2+i \eta_{j}}(\cosh \alpha),
$$

where $\eta_{j}$ and $w_{j}(j=1,2, \ldots, n)$ are the Gauss-Laguerre quadrature points and weights defined in Section 3.1 .

The corresponding temperature distribution is illustrated in Fig. 3a for $\beta_{0}=\pi / 4$. It can be observed that the uniform external heat flux is slightly perturbed by the presence of the insulating inhomogeneity. Fig. 4 a displays the normalized variation of temperature on the spherical surface, $\beta=$ $\beta_{0}$, with the angular coordinate $\theta$ introduced in Fig.1, for various values of $\beta_{0}$. In particular, the solid curve for $\beta_{0}=\pi / 2$ corresponds to a spherical inhomogeneity and it recovers the analytical solution given by $1.5 \cos \theta$ (Maxwell, 1873).

\section{Heat flux orthogonal to the symmetry axis}

We now consider the non-axisymmetric problem corresponding to the heat flux along the $y$-axis orthogonal to the symmetry axis: $\boldsymbol{q}_{0}=\left(0, q_{0}, 0\right)$. Similarly to the previous section, we split the temperature field $T$ into the basic contribution $T_{0}$ induced by the assigned heat flux $\boldsymbol{q}_{0}$ in a homogeneous medium and the correction $T_{1}$ due to the presence of the cavity. In this case, $T_{0}$ is given by

$$
T_{0}(\alpha, \beta)=-\frac{q_{0}}{k} y=-\frac{q_{0} R}{k} \frac{\sin \beta_{0} \sinh \alpha \sin \gamma}{\cosh \alpha-\cos \beta} .
$$

Both fields $T_{0}$ and $T_{1}$ are harmonic and even in $\beta$. The most general harmonic function $T_{1}$ in toroidal coordinates, which is even in $\beta$ and varies with $\sin \gamma$, can be written in the following form (Morse and Feshbach, 1953; Sneddon, 1972) 


$$
T_{1}(\alpha, \beta)=\frac{q_{0} R}{k} \sin \beta_{0} \sqrt{\cosh \alpha-\cos \beta} \sin \gamma \int_{0}^{\infty} u(\tau) P_{-1 / 2+i \tau}^{1}(\cosh \alpha) \cosh \tau \beta \mathrm{d} \tau,
$$

(with the accuracy of arbitrary additive constant defining the reference temperature). In equation (4.2) $P_{-1 / 2+i \tau}^{1}$ is the first order Legendre function of the first kind with complex index (Bateman and Erdelyi, 1953; Lebedev et al., 1965), which is vanishing at infinity. Similarly to the axisymmetric part, we can consider only the upper half-space where $\beta$ is positive. The unknown function $u(\tau)$ must be found by imposing the boundary condition (2.12) at $\beta=\beta_{0}$ :

$$
\begin{aligned}
\frac{\sin \beta_{0}}{2\left(\cosh \alpha-\cos \beta_{0}\right)} & \int_{0}^{\infty} u(\tau) P_{-1 / 2+i \tau}^{1}(\cosh \alpha) \cosh \tau \beta_{0} \mathrm{~d} \tau+\int_{0}^{\infty} \tau u(\tau) P_{-1 / 2+i \tau}^{1}(\cosh \alpha) \sinh \tau \beta_{0} \mathrm{~d} \tau= \\
& =-\frac{\sinh \alpha \sin \beta_{0}}{\left(\cosh \alpha-\cos \beta_{0}\right)^{5 / 2}} .
\end{aligned}
$$

Using the orthogonality relation (Gradshteyn and Ryzhik, 2007)

$$
\int_{0}^{\infty} P_{-1 / 2+i \tau}^{1}(\cosh \alpha) P_{-1 / 2+i \eta}^{1}(\cosh \alpha) \sinh \alpha d \alpha=\frac{1+4 \tau^{2}}{4 \tau \tanh \tau \pi} \delta(\tau-\eta) .
$$

and the result (A.10) derived in the Appendix, one gets the following Fredholm integral equation of the second kind for the unknown function $u(\tau)$

$$
\frac{\sinh \tau \beta_{0}}{\tanh \tau \pi} \frac{1+4 \tau^{2}}{4} u(\tau)+\frac{\sin \beta_{0}}{2} \int_{0}^{\infty} U(\tau, \eta) u(\eta) \cosh \eta \beta_{0} \mathrm{~d} \eta=\frac{\sqrt{2}}{3}\left(1+4 \tau^{2}\right) \frac{\sinh \tau\left(\pi-\beta_{0}\right)}{\sinh \tau \pi}
$$

where the subkernel

$$
U(\tau, \eta)=\int_{0}^{\infty} \frac{P_{-1 / 2+i \tau}^{1}(\cosh \alpha) P_{-1 / 2+i \eta}^{1}(\cosh \alpha)}{\cosh \alpha-\cos \beta_{0}} \sinh \alpha \mathrm{d} \alpha=\int_{1}^{\infty} \frac{P_{-1 / 2+i \tau}^{1}(s) P_{-1 / 2+i \eta}^{1}(s)}{s-\cos \beta_{0}} \mathrm{~d} s,
$$

is a symmetric function and the integral converges since $P_{-1 / 2+i \tau}^{1}(\cosh \alpha)$ decays as $e^{-\alpha / 2}$. The numerical calculation of the subkernel $U(\tau, \eta)$ for transversal heat flux is also addressed in Appendix B. The substitution suggested by Cochran (1972),

$$
v(\tau)=u(\tau) \sqrt{\left(1+4 \tau^{2}\right) \frac{\sinh \tau \beta_{0} \cosh \tau \beta_{0}}{4 \tanh \tau \pi}},
$$

then transforms equation (4.5) into a Fredholm integral equation for the function $v(\tau)$ in the standard form 


$$
v(\tau)+\frac{\sin \beta_{0}}{2} \int_{0}^{\infty} K(\tau, \eta) v(\eta) \mathrm{d} \eta=f(\tau)
$$

where

$$
f(\tau)=\frac{4}{3} \sqrt{\frac{1+4 \tau^{2}}{\sinh 2 \tau \pi \tanh \tau \beta_{0}}} \sinh \tau\left(\pi-\beta_{0}\right),
$$

and

$$
K(\tau, \eta)=\sqrt{\frac{4}{1+4 \tau^{2}} \frac{\tanh \tau \pi}{\tanh \tau \beta_{0}}} U(\tau, \eta) \sqrt{\frac{4}{1+4 \eta^{2}} \frac{\tanh \eta \pi}{\tanh \eta \beta_{0}}},
$$

is the symmetric kernel.

The numerical solution of the integral equation can be calculated by Gauss-Laguerre quadrature rule discussed in Section 3.1.

\subsection{Temperature field}

Once the unknowns $v_{j}$, for $j=1,2, \ldots, n$, have been obtained, the perturbation temperature field follows from (4.2) and (4.7) as

$$
\begin{aligned}
T_{1}(\alpha, \beta)= & \frac{q_{0} R}{k} \sin \beta_{0} \sin \gamma \sqrt{\cosh \alpha-\cos \beta} \times \\
& \int_{0}^{\infty} v(\tau) \sqrt{\frac{4}{1+4 \tau^{2}} \frac{\tanh \tau \pi}{\sinh \tau \beta_{0} \cosh \tau \beta_{0}}} P_{-1 / 2+i \tau}^{1}(\cosh \alpha) \cosh \tau \beta \mathrm{d} \tau,
\end{aligned}
$$

and, thus, the perturbation temperature field on the pore surface at $\beta=\beta_{0}$ is given by

$$
T_{1}\left(\alpha, \beta_{0}\right)=\frac{q_{0} R}{k} \sin \beta_{0} \sin \gamma \sqrt{\cosh \alpha-\cos \beta_{0}} \int_{0}^{\infty} v(\tau) \sqrt{\frac{4}{1+4 \tau^{2}} \frac{\tanh \tau \pi}{\tanh \tau \beta_{0}}} P_{-1 / 2+i \tau}^{1}(\cosh \alpha) d \tau .
$$

The latter integral can be calculated using the Gauss-Laguerre quadrature rule:

$$
T_{1}\left(\alpha, \beta_{0}\right)=\frac{q_{0} R}{k} \sin \beta_{0} \sin \gamma \sqrt{\cosh \alpha-\cos \beta_{0}} \sum_{j=1}^{n} w_{j} v_{j} \sqrt{\frac{4}{1+4 \eta_{j}^{2}} \frac{\tanh \eta_{j} \pi}{\tanh \eta_{j} \beta_{0}}} P_{-1 / 2+i \eta_{j}}^{1}(\cosh \alpha) .
$$

where $\eta_{j}$ and $w_{j}(j=1,2, \ldots, n)$ are the Gauss-Laguerre quadrature points and weights discussed in Section 3.1 .

The corresponding temperature distribution is illustrated in Fig. $3 b$ for $\beta_{0}=\pi / 4$. Fig. $4 b$ displays the normalized variation of temperature on the spherical surface $\beta=\beta_{0}$, with the angular coordinate $\theta$ 
introduced in Fig.1, for various values of $\beta_{0}$. In particular, the solid curve for $\beta_{0}=\pi / 2$ corresponds to a spherical inhomogeneity and it recovers the analytical solution given by $1.5 \sin \theta$ (Maxwell, 1873).

\section{Resistivity contribution tensor.}

The resistivity contribution tensor $\mathbf{R}$ for a pore or insulating inhomogeneity can be calculated combining (1.1) and (1.2):

$$
\mathbf{R} \cdot \mathbf{q}_{0}=\frac{1}{V_{*}} \int_{S} T \boldsymbol{n} \mathrm{d} S
$$

where $\mathbf{q}_{0}$ is the remote heat flux vector, and $V_{1}$ and $S$ are the volume and surface of the pore, respectively. In particular, component $R_{33}$ can be calculated using the axisymmetric solution given in Section 3. The change in temperature gradient due to the insulating inhomogeneity bounded by surface $\beta= \pm \beta_{0}$ is given by the surface integral

$$
\phi=\int_{S} T\left(\alpha, \beta_{0}\right) \boldsymbol{n} \mathrm{d} A,
$$

where $\mathbf{n}=-\mathbf{e}_{\beta}$ is the outer unit normal on the surface. Let $\mathbf{x}$ denote the position vector on the double sphere surface, then using (2.1) one finds:

$$
\text { n } \begin{aligned}
d A & =\frac{\partial \mathbf{x}}{\partial \alpha} \times \frac{\partial \mathbf{x}}{\partial \gamma} \mathrm{d} \alpha \mathrm{d} \gamma= \\
& =\left\{\sinh \alpha \sin \beta_{0} \cos \gamma, \sinh \alpha \sin \beta_{0} \sin \gamma, 1-\cosh \alpha \cos \beta_{0}\right\} \frac{a^{2} \sinh \alpha \mathrm{d} \alpha \mathrm{d} \gamma}{\left(\cosh \alpha-\cos \beta_{0}\right)^{3}}
\end{aligned}
$$

\subsection{Heat flux along the symmetry axis.}

In the case of remote heat flux directed along the symmetry axis of the pore (z-axis), the sole nonvanishing component of vector (5.2) across the upper half-pore is

$$
\phi_{z}=2 \pi R^{2} \sin ^{2} \beta_{0} \int_{0}^{\infty} \frac{1-\cosh \alpha \cos \beta_{0}}{\left(\cosh \alpha-\cos \beta_{0}\right)^{3}} T\left(\alpha, \beta_{0}\right) \sinh \alpha d \alpha .
$$

The substitution of the total temperature field $T=T_{0}+T_{1}$ defined by equations (3.1) and (3.2) into (5.4) yields the total $\phi_{z}=\phi_{z}^{(0)}+\phi_{z}^{(1)}$, where 


$$
\phi_{z}^{(0)}=-2 \pi R^{3} \frac{q_{0}}{k} \sin ^{4} \beta_{0} \int_{0}^{\infty} \frac{1-\cosh \alpha \cos \beta_{0}}{\left(\cosh \alpha-\cos \beta_{0}\right)^{4}} \sinh \alpha d \alpha=-\frac{q_{0}}{k} \pi R^{3} \frac{\left(2-\cos \beta_{0}\right) \sin ^{4} \beta_{0}}{3\left(1-\cos \beta_{0}\right)^{2}},
$$

and

$$
\begin{aligned}
\phi_{z}^{(1)}= & \frac{q_{0}}{k} 2 \pi R^{3} \sin ^{3} \beta_{0} \times \\
& \sum_{j=1}^{n} w_{j} v_{j} \sqrt{\tanh \eta_{j} \pi \tanh \eta_{j} \beta_{0}} \int_{0}^{\infty} \frac{\left(1-\cosh \alpha \cos \beta_{0}\right) \sinh \alpha}{\left(\cosh \alpha-\cos \beta_{0}\right)^{5 / 2}} P_{-1 / 2+i \eta_{j}}(\cosh \alpha) d \alpha .
\end{aligned}
$$

Using (A.5), (5.6) leads to the following expression

$$
\phi_{z}^{(1)}=\frac{q_{0}}{k} \frac{8}{3} \pi R^{3} \sin ^{3} \beta_{0} \sum_{j=1}^{n} w_{j} v_{j} \sqrt{\frac{\tanh \eta_{j} \beta_{0}}{\sinh 2 \eta_{j} \pi}}\left[2 \eta_{j} \cosh \eta_{j}\left(\pi-\beta_{0}\right)-\cot \beta_{0} \sinh \eta_{j}\left(\pi-\beta_{0}\right)\right] .
$$

Then, the axial component of the resistivity contribution tensor is

$$
R_{z z}=\frac{\phi_{z}}{V^{*} q_{0}}
$$

where

$$
V^{*}=\frac{\pi}{3} R^{3}\left(2+3 \cos \beta_{0}-\cos ^{3} \beta_{0}\right)
$$

is the volume of the upper half-cavity.

The variation of the axial component $R_{z z}$ of the resistivity contribution tensor versus the ratio $\beta_{0} / \pi$ is illustrated in Fig. $5 a$. It can be observed that $R_{z z}$ tends to infinity as $\beta_{0}$ tends to $\pi$ (case corresponding to a penny-shaped crack). The contribution to the overall resistivity is still finite since $R_{z z}$ must be multiplied by the vanishing volume of the inhomogeneity. The accuracy of the results get worse as $\beta_{0}$ tends to 0 (two touching spheres) since in the limiting case of $\beta_{0}=0$ the system of toroidal coordinates degenerates, namely $a$ tends to 0 and the circle resulted from intersection of spheres collapses into a point. This case is considered separately in Section 6.

\subsection{Heat flux orthogonal to the symmetry axis.}

In the case of heat flux directed along the $y$-axis, the sole non-vanishing component of vector (5.2) across the upper half-cavity is

$$
\phi_{y}=R^{2} \sin ^{3} \beta_{0} \int_{0}^{\infty} \frac{\sinh ^{2} \alpha}{\left(\cosh \alpha-\cos \beta_{0}\right)^{3}} \mathrm{~d} \alpha \int_{0}^{2 \pi} T\left(\alpha, \beta_{0}\right) \sin \gamma \mathrm{d} \gamma .
$$


The substitution of the total temperature field $T=T_{0}+T_{1}$ defined by equations (4.1) and (4.2) into (5.10) yields the total $\phi_{y}=\phi_{y}{ }^{(0)}+\phi_{y}{ }^{(1)}$, where

$$
\phi_{y}(0)=-\frac{q_{0}}{k} \pi R^{3} \sin ^{4} \beta_{0} \int_{0}^{\infty} \frac{\sinh ^{3} \alpha}{\left(\cosh \alpha-\cos \beta_{0}\right)^{4}} \mathrm{~d} \alpha=-\frac{q_{0}}{k} \pi R^{3} \frac{\left(2-\cos \beta_{0}\right) \sin ^{4} \beta_{0}}{3\left(1-\cos \beta_{0}\right)^{2}},
$$

and

$$
\phi_{y}{ }^{(1)}=\frac{q_{0}}{k} \pi R^{3} \sin ^{4} \beta_{0} \sum_{j=1}^{n} w_{j} v_{j} \sqrt{\frac{4}{1+4 \eta_{j}^{2}} \frac{\tanh \eta_{j} \pi}{\tanh \eta_{j} \beta_{0}}} \int_{0}^{\infty} \frac{P_{-1 / 2+i \eta_{j}}^{1}(\cosh \alpha) \sinh ^{2} \alpha}{\left(\cosh \alpha-\cos \beta_{0}\right)^{5 / 2}} \mathrm{~d} \alpha .
$$

By using the result (A.10) for $\tau=\eta_{j}$ and $\beta=\beta_{0}$, from (5.12) one gets

$$
\phi_{y}{ }^{(1)}=-\frac{4}{3} \frac{q_{0}}{k} \pi R^{3} \sin ^{3} \beta_{0} \sum_{j=1}^{n} w_{j} v_{j} \sqrt{\frac{1+4 \eta_{j}^{2}}{\sinh 2 \eta_{j} \pi \tanh \eta_{j} \beta_{0}}} \sinh \eta_{j}\left(\pi-\beta_{0}\right) .
$$

Therefore, in plane components of the resistivity contribution tensor are

$$
R_{y y}=R_{x x}=\frac{\phi_{y}}{V^{*} q_{0}}
$$

where $V^{*}$ is the volume of the upper half-cavity defined in (5.9).

The variation of the in plane component $R_{x x}=R_{y y}$ of the resistivity contribution tensor versus the ratio $\beta_{0} / \pi$ is illustrated in Fig. $5 b$. It can be observed that $R_{x x}$ tends to $1 / k$ as $\beta_{0}$ tends to $\pi$ (pennyshaped crack) and, thus, no contribution comes from that kind of inhomogeneities to the effective resistivity.

\subsection{Approximation by spheroidal pores or insulators}

Let us now compare the results obtained in (5.1) and (5.2) with those available for oblate and prolate spheroidal insulating inhomogeneities of the aspect ratio $\rho=b_{s} / a_{s}$ (see, for example, Kachanov and Sevostianov, 2018), where $a_{s}$ is the radius of the spheroid and $b_{s}$ is the semi-axis of the spheroid along the $z$-axis, namely

$$
k R_{x x}=k R_{y y}=\frac{1}{1-f_{0}}, \quad R_{z z}=\frac{1}{2 f_{0}},
$$

where 


$$
f_{0}=\frac{\rho^{2}(1-g)}{2\left(\rho^{2}-1\right)}, \quad g(\rho)= \begin{cases}\frac{1}{\rho \sqrt{1-\rho^{2}}} \arctan \frac{\sqrt{1-\rho^{2}}}{\rho}, & \text { oblate shape }(\rho<1) \\ \frac{1}{2 \rho \sqrt{\rho^{2}-1}} \ln \frac{\rho+\sqrt{\rho^{2}-1}}{\rho-\sqrt{\rho^{2}-1}}, & \text { prolate shape }(\rho>1)\end{cases}
$$

The components of the resistivity contribution tensor for an insulating inhomogeneity formed by two coalesced spheres can be approximated with good accuracy by the corresponding components calculated for an insulating spheroid that has the same volume and the same outer radius and, thus, the following aspect ratio

$$
\rho=\frac{R^{3}}{2 a_{s}^{3}}\left(2+3 \cos \beta_{0}-\cos ^{3} \beta_{0}\right),
$$

where

$$
a_{s}= \begin{cases}R, & \text { for } \beta_{0} \leq \pi / 2 \\ R \sin \beta_{0}, & \text { for } \quad \beta_{0}>\pi / 2 .\end{cases}
$$

Therefore, the two coalesced pores can be approximated by a prolate spheroid for $\beta_{0} \leq \pi / 2$ and by an oblate spheroid for $\beta_{0}>\pi / 2$ as soon as the resistivity contribution tensor is considered. The variations of the components of resistivity contribution tensor for the approximating spheroids with $\beta_{0}$ are plotted in Fig. 5 by dashed lines. These curves are very close to the results obtained for the two coalesced insulating spheres and coincide with them for $\beta_{0}=\pi / 2$ and $\beta_{0}=\pi$. The special case of an insulating spherical pore is recovered for $\beta_{0}=\pi / 2$. In this case, the aspect ratio $\rho$ tends to 1 , so that $g=$ 1 and $f_{0}=1 / 3$ and, thus, according to (5.15) the components of resistivity contribution tensor are $R_{x x}=$ $R_{y y}=R_{z z}=1.5 / k$, in agreement with the results shown in Fig. 5 . The limiting case of the penny-shaped crack is recovered as $\beta_{0}$ tends to $\pi$. Then, the aspect ratio $\rho$ tends to 0 and thus $g$ tends to $\pi /(2 \rho), g$ becomes unbounded, and $f_{0}$ tends to 0 . In this case, equation (5.15) yields $R_{x x}=R_{y y}=1 / k$ and $R_{z z}$ becomes unbounded, in agreement with the results presented in Fig. 5.

Regardless of the monotonic variation with $\beta_{0}$ exhibited by the components of resistivity contribution tensor of spheroids in Fig. 5 (dashed curves), those of the double sphere pore display a non-monotonic variation (solid curves). The components $R_{z z}$ and $R_{y y}$ attain indeed a stationary value at $\beta_{0}=0$. However, $R_{z z}$ displays a minimum at about $\beta_{0}=\pi / 4$ and $R_{y y}$ reaches a maximum at about $\beta_{0}=$ $\pi / 5$ (see Fig. 6); thus, the extrema of the resistivity contribution tensor components do not occur for $\beta_{0}$ $=0$, as it happens for spheroids (see Fig. 5). 
Combining our results with the computational data of Sevostianov et al (2014), we can plot the components of the resistivity contribution tensor as functions of the distance between the pore centres shown in Fig. 6. We assume that one spherical pore is fixed (i.e. origin of the coordinate system is located at the pore centre). The second pore is located at certain distance from the first one. If this distance is large enough, the pores do not see each other and their resistivity contribution tensor coincides with the one for a single sphere. When distance decreases, the magnitudes of the resistivity contribution tensor components is growing up reaching their maxima a bit after the spheres start coalescing. Then, further pores coalescence process decreases the absolute values of the components down to that for a single sphere. After that the collapsing of a single pore corresponds to the "negative distance" when the centre of the second pore moves to the left of the origin of coordinates. In the limiting case, we obtain the values corresponding to a penny shaped crack.

In Fig. 7, we observe that the minimum of $R_{z z}$ and the maximum of $R_{y y}$ occur at $z_{c} / R=1.6$ and 1.7, respectively (when the two spheres are coalesced), in agreement with Fig. 6.

\section{Limiting case of two touching spheres}

In this section, we derive the expressions for the resistivity contribution tensor components in the limiting case of two touching spheres. In this case, the circle resulted from intersection of spheres collapses into a point (origin of the coordinates) $\beta_{0} \rightarrow 0$ and the solutions of the integral equations (3.5) and (4.5) turn out to be singular. Due to that, we use the tangent sphere coordinate system $(\xi, \eta, \gamma)$ sketched in Figure 8 (see Moon and Spencer, 1961). Coordinate surfaces of this system are formed by toroids and spheres tangent to the $x y$ coordinate plane at the origin. Coordinates of the tangent sphere coordinate system are related to the Cartesian coordinates by the following relations

$$
x=\frac{2 R \eta}{\xi^{2}+\eta^{2}} \cos \gamma, \quad y=\frac{2 R \eta}{\xi^{2}+\eta^{2}} \sin \gamma, \quad z=\frac{2 R \xi}{\xi^{2}+\eta^{2}} .
$$

The surface of the two touching spheres of radius $R$ with the centres laying on $z$-axis are defined by the coordinate $\xi= \pm 1$, for $0 \leq \eta<\infty$ and $0 \leq \gamma<2 \pi$.

The temperature distribution $T$ under steady-state heat flux must satisfy the Laplace equation (2.3) and the Neumann boundary condition at the pore surface:

$$
\frac{\partial T}{\partial \xi}=0, \quad \text { at } \xi= \pm 1 .
$$


Far from the pore (both $\xi$ and $\eta$ tend to zero) the heat flux must approach the remotely applied one $\boldsymbol{q}=\boldsymbol{q}_{0}$.

We now calculate the temperature field and the components of the resistivity contribution tensor for axial and transverse heat flux for $\beta_{0}=0$. Note that the solution below differ from the one of Pitkonen (2008) by the boundary condition at infinity. To construct the resistivity contribution tensor, we need thermal flux rather than temperature to be prescribed.

\subsection{Heat flux along the symmetry axis.}

For $\boldsymbol{q}_{0}=\left(0,0, q_{0}\right)$, we split the temperature field onto the temperature $T_{0}$ induced by the uniform heat flux in a homogeneous medium and the perturbation temperature field $T_{1}$ due to the presence of the inhomogeneity as follows

$$
\begin{aligned}
& T_{0}(\xi, \eta)=-\frac{q_{0}}{k} z=-\frac{q_{0} R}{k} \frac{2 \xi}{\xi^{2}+\eta^{2}} \\
& T_{1}(\xi, \eta)=\frac{q_{0} R}{k} \sqrt{\xi^{2}+\eta^{2}} \int_{0}^{\infty} u(s) \sinh s \xi J_{0}(s \eta) \mathrm{d} s, \quad-1 \leq \xi \leq 1
\end{aligned}
$$

where $J_{0}$ is the Bessel function of the first kind of zero order, which is vanishing at infinity. Function $T_{1}$ in (6.4) is the most general harmonic axisymmetric function in the tangent sphere coordinates, which is odd in $\xi$ (Moon and Spencer, 1961). The unknown function $u(\tau)$ must be found by introducing (6.3) and (6.4) into the boundary condition (6.2) at $\xi=1$ :

$$
\frac{2\left(1-\eta^{2}\right)}{\left(1+\eta^{2}\right)^{3 / 2}}+\int_{0}^{\infty} u(s)(\sinh s+s \cosh s) J_{0}(s \eta) \mathrm{d} s+\eta^{2} \int_{0}^{\infty} u(s) s \cosh s J_{0}(s \eta) \mathrm{d} s=0 .
$$

Using connection between Bessel function $J_{0}$ and its derivative (Gradshteyn and Ryzhik, 2007)

$$
J_{0}(s \eta)=-\frac{1}{s \eta^{2}} \frac{d}{d s}\left[s \frac{\mathrm{d} J_{0}(s \eta)}{\mathrm{d} s}\right]
$$

and integrating twice the last integral in (6.5) by parts, we obtain

$$
\begin{aligned}
& \frac{2\left(1-\eta^{2}\right)}{\left(1+\eta^{2}\right)^{3 / 2}}+\int_{0}^{\infty} u(s)(\sinh s+s \cosh s) J_{0}(s \eta) \mathrm{d} s-\int_{0}^{\infty} \frac{\mathrm{d}}{\mathrm{d} s}\left[s \frac{\mathrm{d}}{\mathrm{d} s}\{u(s) \cosh s\}\right] J_{0}(s \eta) \mathrm{d} s+ \\
& {\left[s \frac{d}{d s}\{u(s) \cosh s\} J_{0}(s \eta)-u(s) s \cosh s \frac{d J_{0}(s \eta)}{d s}\right]_{0}^{\infty}=0 .}
\end{aligned}
$$


Then, using the following definite integrals (Gradshteyn and Ryzhik, 2007) in the first term of (6.7)

$$
\int_{0}^{\infty} e^{-s} J_{0}(s \eta) \mathrm{d} s=\frac{1}{\sqrt{1+\eta^{2}}}, \quad \int_{0}^{\infty} s e^{-s} J_{0}(s \eta) \mathrm{d} s=\frac{1}{\left(1+\eta^{2}\right)^{3 / 2}},
$$

the following ordinary differential equation can be derived for the unknown function $u(s)$

$$
\frac{d}{d s}\left[s \frac{d}{d s}\{u(s) \cosh s\}\right]-(\sinh s+s \cosh s) u(s)=2(2 s-1) e^{-s},
$$

Or, equivalently,

$$
s \cosh s u^{\prime \prime}(s)+(2 s \sinh s+\cosh s) u^{\prime}(s)=2(2 s-1) e^{-s} .
$$

The latter equation can be integrated to

$$
u^{\prime}(s)=2 \tanh s-2+\frac{s}{\cosh ^{2} s}+\frac{C_{1}}{s \cosh ^{2} s},
$$

Constant $C_{1}$ can be determined from the condition $u(0)=0$, required for vanishing of the perturbation flux at infinity, which yields $C_{1}=0$. Further integration of (6.11) gives

$$
u(s)=\ln (\cosh s)+s(\tanh s-2)+C_{2} .
$$

Constant $C_{2}$ can be determined by imposing the condition $u(s) \rightarrow 0$ as $s \rightarrow \infty$. which follows from the request that the integral in (6.4) be convergent; it yields $C_{2}=\ln 2$. Thus, the solution $u(s)$ is completely determined as follows

$$
u(s)=\ln (2 \cosh s)+s(\tanh s-2) .
$$

The corresponding temperature distribution is shown in Figure 9a. The variation of the temperature on the sphere surface with the angular coordinate $\theta$ is plotted in Fig. 4a (solid black line).

We can now calculate the temperature flux on the pore surface. If $\mathbf{x}$ denote the position vector on the sphere surface, then (6.1) yields:

$$
\mathbf{n} d A=\left[\frac{\partial \mathbf{x}}{\partial \eta} \times \frac{\partial \mathbf{x}}{\partial \gamma}\right]_{\xi=1} \mathrm{~d} \eta \mathrm{d} \gamma=\frac{8 R^{2} \eta}{\left(1+\eta^{2}\right)^{3}}\left\{\eta \cos \gamma, \eta \sin \gamma, 1-\eta^{2}\right\} \mathrm{d} \eta \mathrm{d} \gamma .
$$

Therefore, the non-vanishing component of heat flux vector (5.2) across the upper half-pore for remote heat flux directed along the symmetry axis is

$$
\phi_{z}=8 \pi R^{2} \int_{0}^{\infty} \frac{\eta\left(1-\eta^{2}\right)}{\left(1+\eta^{2}\right)^{3}} T(1, \eta) \mathrm{d} \eta .
$$

The substitution of the total temperature field $T=T_{0}+T_{1}$ defined by equations (6.2) and (6.3) into (6.15), yields the heat flux $\phi_{z}=\phi_{z}^{(0)}+\phi_{z}^{(1)}$ with 


$$
\phi_{z}^{(0)}=-32 \frac{q_{0}}{k} \pi R^{3} \int_{0}^{\infty} \frac{\eta\left(1-\eta^{2}\right)}{\left(1+\eta^{2}\right)^{4}} \mathrm{~d} \eta=-\frac{q_{0}}{k} \frac{4}{3} \pi R^{3},
$$

and

$$
\begin{aligned}
\phi_{z}^{(1)} & =8 \frac{q_{0}}{k} \pi R^{2} \int_{0}^{\infty}[\ln (2 \cosh s)+s(\tanh s-2)] \sinh s \mathrm{~d} s \int_{0}^{\infty} \frac{\eta\left(1-\eta^{2}\right)}{\left(1+\eta^{2}\right)^{3}} J_{0}(s \eta) \mathrm{d} \eta= \\
& =\frac{8}{3} \frac{q_{0}}{k} \pi R^{2} \int_{0}^{\infty}[\ln (2 \cosh s)+s(\tanh s-2)](2 s-1) e^{-s} \sinh s d s=\frac{q_{0}}{k} \frac{8-9 \zeta(3)}{6} \pi R^{2},
\end{aligned}
$$

where $\zeta$ denotes the Riemann zeta function. Then, the axial component of the resistivity contribution tensor is

$$
R_{z z}=\frac{\phi_{z}}{V^{*} q_{0}}=-\frac{9}{8 k} \zeta(3)=-\frac{1.3523}{k},
$$

where

$$
V^{*}=\frac{4}{3} \pi R^{3}
$$

is the volume of the upper spherical cavity.

\subsection{Heat flux orthogonal to the symmetry axis.}

For $\boldsymbol{q}_{0}=\left(0, q_{0}, 0\right)$ the temperature field $T$ can be split into

$$
\begin{aligned}
& T_{0}(\xi, \eta)=-\frac{q_{0}}{k} y=-\frac{q_{0} R}{k} \frac{2 \eta}{\xi^{2}+\eta^{2}} \sin \gamma, \\
& T_{1}(\xi, \eta)=\frac{q_{0} R}{k} \sin \gamma \sqrt{\xi^{2}+\eta^{2}} \int_{0}^{\infty} u(s) \cosh s \xi J_{1}(s \eta) \mathrm{d} s,
\end{aligned}
$$

for $-1 \leq \xi \leq 1$, where $J_{1}$ is the Bessel function of the first kind of first order, which vanishes at infinity. Function $T_{1}$ in (6.21) is the most general harmonic function in the tangent sphere coordinates, which varies as $\sin \gamma$ and is even in $\xi$. The unknown function $u(\tau)$ must be found by introducing (6.20) and (6.21) in the boundary condition (6.2) at $\xi=1$ :

$$
\frac{4 \eta}{\left(1+\eta^{2}\right)^{3 / 2}}+\int_{0}^{\infty} u(s)(\cosh s+s \sinh s) J_{1}(s \eta) \mathrm{d} s+\eta^{2} \int_{0}^{\infty} u(s) s \sinh s J_{1}(s \eta) \mathrm{d} s=0 .
$$

Using connection between Bessel function $J_{1}$ and its derivative (Gradshteyn and Ryzhik, 2007) 


$$
\begin{aligned}
& J_{1}(s \eta)=-\frac{s}{s^{2} \eta^{2}-1} \frac{\mathrm{d}}{\mathrm{d} s}\left[s \frac{\mathrm{d} J_{1}(s \eta)}{\mathrm{d} s}\right] \\
& \int_{0}^{\infty} s e^{-s} J_{1}(s \eta) \mathrm{d} s=\frac{\eta}{\left(1+\eta^{2}\right)^{3 / 2}},
\end{aligned}
$$

and integrating twice the last integral in (6.22) by parts, we obtain

$$
\int_{0}^{\infty}\left\{-\frac{d}{d s}\left[s \frac{d}{d s}(u(s) \sinh s)\right]+u(s)\left(\cosh s+s \sinh s+\frac{\sinh s}{s}\right)+4 s e^{-s}\right\} J_{1}(s \eta) \mathrm{d} s=0 .
$$

so that

$$
\begin{aligned}
\eta^{2} \int_{0}^{\infty} u(s) s \sinh s J_{1}(s \eta) \mathrm{d} s=\int_{0}^{\infty} u(s) \sinh s \frac{s^{2} \eta^{2}-1+1}{s} J_{1}(s \eta) \mathrm{d} s= \\
=\int_{0}^{\infty} u(s) \sinh s\left\{\frac{J_{1}(s \eta)}{s}-\frac{\mathrm{d}}{\mathrm{d} s}\left[s \frac{\mathrm{d} J_{1}(s \eta)}{\mathrm{d} s}\right]\right\} \mathrm{d} s= \\
=\int_{0}^{\infty}\left\{s \frac{\mathrm{d}}{\mathrm{d} s}[u(s) \sinh s] \frac{\mathrm{d} J_{1}(s \eta)}{\mathrm{d} s}+u(s) \sinh s \frac{J_{1}(s \eta)}{s}\right\} \mathrm{d} s-\left[u(s) s \sinh s \frac{d J_{1}(s \eta)}{d s}\right]_{0}^{\infty}= \\
=\int_{0}^{\infty}\left\{-\frac{\mathrm{d}}{\mathrm{d} s}\left[s \frac{\mathrm{d}}{\mathrm{d} s}(u(s) \sinh s)\right]+u(s) \frac{\sinh s}{s}\right\} J_{1}(s \eta) \mathrm{d} s \\
\quad+\left[s \frac{\mathrm{d}}{\mathrm{d} s}\{u(s) \sinh s\} J_{1}(s \eta)-u(s) s \sinh s \frac{\mathrm{d} J_{1}(s \eta)}{\mathrm{d} s}\right]_{0}^{\infty}
\end{aligned}
$$

where the last term within the brackets vanishes due to the request for the improper integral in (6.21) be convergent. Then, function $u(s)$ must behave as $o\left(s^{-1 / 2} e^{-s}\right)$ as $s \rightarrow \infty$. Therefore, the following ordinary differential equation is derived from (6.25) for function $u(s)$

$$
\frac{\mathrm{d}}{\mathrm{d} s}\left[s \frac{\mathrm{d}}{\mathrm{d} s}\{u(s) \cosh s\}\right]-\left(\cosh s+s \sinh s+\frac{\sinh s}{s}\right) u(s)=4 s e^{-s}
$$

or, equivalently (Davis, 1977)

$$
s v^{\prime \prime}(s)-v^{\prime}(s)-(s+\operatorname{coth} s) v(s)=4 s^{2} e^{-s},
$$

where

$$
v(s)=u(s) s \sinh s .
$$

Equation (6.28) can be integrated numerically by imposing the boundary conditions 


$$
v(0)=0, \quad \lim _{s \rightarrow \infty} v(s)=0,
$$

necessary for the integral in (6.21) to be convergent.

No explicit closed form solution for (6.28) can be found. Davis (1977) performed a numerical integration of (6.28) following the approach developed by O'Neill (1969) for the motion of two equal touching spheres in a viscous fluid. In order to start the numerical integration at a finite small value of $s$, Davis (1977) worked out a preliminary series representation of the particular integral and complementary function solutions of (6.28) for small values of $s$. He also found that $v(s)$ behaves as $-s^{2} e^{-s}$ for large $s$.

The temperature distribution in the material is shown in Figure 9b. The exact variation of the temperature on the sphere surface with the angular coordinate $\theta$ obtained by numerical integration of (6.28) is plotted in Fig. $4 b$ (solid black line).

The non-vanishing component of the heat flux vector (5.2) across the upper half-cavity is

$$
\phi_{y}=8 R^{2} \int_{0}^{\infty} \frac{\eta^{2}}{\left(1+\eta^{2}\right)^{3}} \mathrm{~d} \eta \int_{0}^{2 \pi} T\left(\alpha, \beta_{0}\right) \sin \gamma \mathrm{d} \gamma .
$$

The substitution of the total temperature field $T=T_{0}+T_{1}$ given by equations (6.20) and (6.21) into (6.31), yields the heat flux $\phi_{y}=\phi_{y}{ }^{(0)}+\phi_{y}{ }^{(1)}$, with

$$
\phi_{y}{ }^{(0)}=-16 \frac{q_{0}}{k} \pi R^{3} \int_{0}^{\infty} \frac{\eta^{3}}{\left(1+\eta^{2}\right)^{4}} \mathrm{~d} \eta=-\frac{q_{0}}{k} \frac{4}{3} \pi R^{3},
$$

and

$$
\phi_{y}(1)=8 \frac{q_{0}}{k} \pi R^{3} \int_{0}^{\infty} u(s) \cosh s \mathrm{~d} s \int_{0}^{\infty} \frac{\eta^{2}}{\left(1+\eta^{2}\right)^{5 / 2}} J_{1}(s \eta) \mathrm{d} \eta=\frac{8}{3} \frac{q_{0}}{k} \pi R^{3} \int_{0}^{\infty} u(s) s e^{-s} \cosh s \mathrm{~d} s .
$$

The numerical calculation of the definite integral in (6.33) is performed using the numerical solution of (6.28), obtained by the procedure proposed by Davis (1971) and Cox and Cooker (2000). The result is

$$
\phi_{y}^{(1)}=-0.8295 \frac{q_{0}}{k} \pi R^{3} .
$$

Therefore, the transversal component of the resistivity contribution tensor is

$$
R_{y y}=\frac{\phi_{y}}{V^{*} q_{0}}=-\frac{1.6221}{k}
$$


where $V^{*}$ is the volume of the upper spherical cavity defined in (6.19).

An approximate analytical result, which may be useful for validating the numerical solution, can be achieved by assuming $v(s) \cong-s^{2} e^{-s}$. Cox and Cooker (2000) noticed indeed that this approximation holds true for the entire range of $s$, not only for large $s$. Correspondingly, from (6.29) it follows

$$
u(s) \cong-\frac{s e^{-s}}{\sinh s},
$$

and the perturbation heat flux on the spherical surface then is

$$
\phi_{y}{ }^{(1)} \cong-\frac{q_{0}}{k} \frac{8}{3} \pi R^{3} \int_{0}^{\infty} s^{2} e^{-2 s} \operatorname{coth} s \mathrm{~d} s=-\frac{q_{0}}{k} \frac{4 \zeta(3)-2}{3} \pi R^{3}=-0.9361 \frac{q_{0}}{k} \pi R^{3} .
$$

The corresponding approximated transversal component of the resistivity contribution tensor is

$$
R_{y y}=\frac{\phi_{y}}{V^{*} q_{0}} \cong-\frac{1.7021}{k}
$$

which is about $5 \%$ larger than the exact result (6.35).

\section{Concluding remarks.}

We solved analytically the problem on distribution of temperature field around a pore or insulating inhomogeneity formed by two overlapping spheres embedded in an isotropic 3-D space subjected to an arbitrarily oriented steady heat flux at infinity. The limiting case of two touching spheres is analyzed separately. The solution is obtained in the form of convergent integrals that can be calculated using the Gauss-Laguerre quadrature rule. The obtained solution is used to construct resistivity contribution tensor for insulating inhomogeneity that has a shape of overlapped spheres. This tensor describes the extra temperature gradient due to the presence of the inhomogeneity. It can be approximated with good accuracy by one for a prolate or oblate spheroidal insulator. Combining our calculations with numerical data of Sevostianov et al. (2014) we show how the resistivity contribution tensor changes when two spherical pores become closer to each other, than coalesce and, after complete coalescence collapse to a circular crack. The resistivity contribution tensor is the key quantity for calculation of the effective properties of the material. The obtained results can be reformulated for the electric conductivity and for the diffusion process (in the electric conductivity problem, temperature and heat flux should be replaced by electric potential and electric current; in the diffusion problem they should be replaced by concentration and diffusion flux). 


\section{Acknowledgement.}

LL gratefully acknowledges financial support from the Italian Ministry of Education, University and Research (MIUR) in the framework of the Project PRIN "Modelling of constitutive laws for traditional and innovative building materials" (code 2017HFPKZY).

ER and IS gratefully acknowledge support from the POR FESR 2014-2020 project IMPReSA -

"Impiego di Materiali Plastici di Riciclo per malte e calcestruzzi Strutturali Alleggeriti" (CUP. E81F18000310009). 


\section{References}

Abramowitz, M., Stegun, I. A. (Eds.). Handbook of Mathematical Functions with Formulas, Graphs, and Mathematical Tables, 9th printing. Dover, New York, 1972.

Argatov, I., Sevostianov, I. (2011) Rigid toroidal inhomogeneity in an elastic medium. International Journal of Engineering Science, 49, 61-74.

Bateman, H., Erdelyi, A. (1953) Higher transcendental functions. Vol.1. New York: McGraw-Hill.

Chowdhury, A., Christov, C. I. (2010) Fast Legendre spectral method for computing the perturbation of a gradient temperature field in an unbounded region due to the presence of two spheres. Numerical Methods in Partial Differential Equations, 26, 1125-1145.

Cochran, J.A. The analysis of linear integral equations. McGraw-Hill Series in Modern Applied Mathematics. New York, 1972.

Cox, S.J., Cooker, M.J. (2000) Potential flow past a sphere touching a tangent plane. Journal of Engineering Mathematics, 38, 355-370.

Davis, A.M.J. (1977). High frequency limiting virtual-mass coefficients of heaving half-immersed spheres. Journal of Fluid Mechanics, 80(2), 305-319.

Felderhof, B.U., Palaniappan, D. (1999) Electrostatic capacitance of two unequal overlapping spheres and the rate of diffusion-controlled absorption. Journal of Applied Physics, 86, 6501-6506.

Felderhof, B.U., Palaniappan, D. (2000) Longitudinal and transverse polarizability of the conducting double sphere. Journal of Applied Physics, 88, 4947-4953.

Gradshteyn, I.S., Ryzhik, I.M. (2007) Table of Integrals, Series, and Products. Elsevier.

Jeffery, G.B. (1912) On a form of the solution of Laplace's equation suitable for problem relating to two spheres, Proc. Roy. Soc. L., A 87, 109-120.

Kachanov, M., Sevostianov, I. (2012) Rice's internal variables formalism and its implications for the elastic and conductive properties of cracked materials, and for the attempts to relate strength to stiffness, Journal of Applied Mechanics, 79, 031002-1-10.

Kachanov, M., Sevostianov, I. (2018) Micromechanics of Materials, with Applications. Springer, 2018.

Krasnitckii, S., Trofimov, A., Radi, E., Sevostianov, I. (2019) Effect of a rigid toroidal inhomogeneity on the elastic properties of a composite. Mathematics and Mechanics of Solids, 24, 1129-1146.

Lanzoni L., Radi E., Sevostianov I. (2018). Effect of cylindrical fibers, with cross-sections formed by two circular arcs, on the overall conductivity of a composite. International Journal of Solids and Structures, 138, 264-276. 
Latta, G. E., Hess, G. B. (1973) Potential flow past a sphere tangent to a plane. Phys. Fluids 16, 974976.

Lebedev, N.N., Silverman, R.A. Special functions and their applications. Courier Dover Publications, Mineola, NY, USA, 1972.

Lebedev, N.N., Skalskaya. I.P., Uflyand, Y.S. Problems of mathematical physics. Prentice-Hall Inc., Englewood Cliff, N.J., USA, 1965.

Liemert, A. (2014) Explicit solution for the electrostatic potential of the conducting double sphere. Journal of Applied Physics, 115, 164907.

Love, J.D. (1975) Dielectric sphere-sphere and sphere-plane problems in electrostatics, The Quarterly Journal of Mechanics and Applied Mathematics, 28, 449-471.

Maxwell, J. C. (1873) A Treatise on Electricity and Magnetism. Clarendon Press, Oxford.

Miloh, T. (1991) On the oblique water-entry problem of a rigid sphere. Journal of Engineering Mathematics, 25, 77-92.

Morse, P.M., Feshbach, H. Methods of theoretical physics, Vol. 1. McGraw-Hill, New York, USA, 1953.

O'Neill M. E. (1969) On asymmetrical slow viscous flows caused by the motion of two equal spheres almost in contact. Proc. Camb. Phil. Soc., 65, 543-556.

Olevsky, E.A. (1998) Theory of sintering: from discrete to continuum. Materials Science and Engineering, R23, 41-100.

Palaniappan, D. (2012) Analytic solutions for some basic problems in electricity involving intersecting sphere and circular cylinder pairs. Electrical Engineering, 94, 107-116.

Pitkonen, M. (2008) Polarizability of a pair of touching dielectric spheres. Journal of Applied Physics 103, 104910.

Pitkonen, M. (2006) Polarizability of the dielectric double-sphere. Journal of Mathematical Physics, 47, 102901.

Pitkonen, M. (2007) An explicit solution for the electric potential of the asymmetric dielectric double sphere. Journal of Physics D: Applied Physics, 40, 1483-1488.

Porollo, S. I., Dvoriashin, A.M., Konobeev, Yu.V., Ivanov, A.A., Shulepin, S.V., and Garner, F.A. (2006) Microstructure and mechanical properties of austenitic stainless steel 12X18H9T after neutron irradiation in the pressure vessel of BR-10 fast reactor at very low dose rates, J. Nucl. Mater. 359, 41-49. 
Radi, E., Sevostianov, I. (2016) Toroidal insulating inhomogeneity in an infinite space and related problems, Proceedings of the Royal Society of London - A: Mathematical, Physical \& Engineering Sciences, A472, 20150781.

Schiffer, M., Szego, G. (1949) Virtual mass and polarization. Transactions of the American Mathematical Society, 67, 130-205.

Sevostianov, I., Kachanov, M. (2002) Explicit cross-property correlations for anisotropic two-phase composite materials. J. Mech. Phys. Solids, 50, 253-282.

Sevostianov, I., Kachanov, M. (2015) On the possibility to represent effective properties of a material with inhomogeneities in terms of a single concentration parameter. International Journal of Solids and Structures 52, 197-204.

Sevostianov, I., Kachanov, M., Drach, B. (2014) On the effect of interactions of inhomogeneities on the overall elastic and conductive properties. International Journal of Solids and Structures, 51, 4531-4543.

Sneddon, I. H. The use of integral transforms. McGraw-Hill, New York, USA, 1972.

Stoy, R.D. (1986a) Solution procedure for the Laplace equation in bispherical coordinates for two spheres in a uniform external field: Parallel orientation. Journal of Applied Physics, 65, 2611-2615.

Stoy, R.D. (1986b) Solution procedure for the Laplace equation in bispherical coordinates for two spheres in a uniform external field: Perpendicular orientation. Journal of Applied Physics, 66, 50935095.

Tong, G.P. (1996) Electrostatics of two conducting spheres intersecting at angles. European Journal of Physics, 17, 244-249.

Witze, C. P., Schrock, V. E., Chambré, P. L. (1968). Flow about a growing sphere in contact with a plane surface. International Journal of Heat and Mass Transfer, 11(11), 1637-1652.

Zhou, H., Li, C., Yang., H., Luo, X., Yang, G., Li, W., Hussain, T., Li, C. (2019) Pores structure change induced by heat treatment in cold-sprayed Ti6A14V coating. Journal of Thermal Spray Technology, 28, 1199-1211. 


\section{Appendix A. Useful definite integrals}

Let us calculate some definite integrals used in deriving eqns (3.5) and (5.6). Let us start from the following definite integral, which can be found in Liemert (2014) eqn (12):

$$
\int_{0}^{\infty} \frac{P_{-1 / 2+i \tau}(\cosh \alpha)}{(\cosh \alpha-\cos \beta)^{1 / 2}} \sinh \alpha d \alpha=\sqrt{2} \frac{\cosh \tau(\pi-|\beta|)}{\tau \sinh \tau \pi} .
$$

By taking the derivative with respect to $\beta$ of eqn (A.1) one gets

$$
\int_{0}^{\infty} \frac{P_{-1 / 2+i \tau}(\cosh \alpha)}{(\cosh \alpha-\cos \beta)^{3 / 2}} \sinh \alpha d \alpha=2 \sqrt{2} \frac{\sinh \tau(\pi-|\beta|)}{\sin |\beta| \sinh \tau \pi} .
$$

By taking the derivative with respect to $\beta$ of eqn (A.2) one gets

$$
\int_{0}^{\infty} \frac{P_{-1 / 2+i \tau}(\cosh \alpha)}{(\cosh \alpha-\cos \beta)^{5 / 2}} \sinh \alpha d \alpha=\frac{4 \sqrt{2}}{3} \frac{\cot |\beta| \sinh \tau(\pi-|\beta|)+\tau \cosh \tau(\pi-|\beta|)}{\sin ^{2} \beta \sinh \tau \pi} .
$$

Then by using (A.3) and the result

$$
\int_{0}^{\infty} P_{-1 / 2+i \tau}(\cosh \alpha) \frac{\cosh \alpha \cos \beta-1-(\sin \beta)^{2} / 2}{(\cosh \alpha-\cos \beta)^{5 / 2}} \sinh \alpha d \alpha=-\frac{2 \sqrt{2} \tau}{\sinh \tau \pi} \cosh \tau(\pi-|\beta|),
$$

one obtains

$$
\begin{aligned}
& \int_{0}^{\infty} \frac{\cosh \alpha \cos \beta-1}{(\cosh \alpha-\cos \beta)^{5 / 2}} P_{-1 / 2+i \tau}(\cosh \alpha) \sinh \alpha d \alpha= \\
& \quad=\frac{2 \sqrt{2}}{3 \sinh \tau \pi}[\cot |\beta| \sinh \tau(\pi-|\beta|)-2 \tau \cosh \tau(\pi-|\beta|)] .
\end{aligned}
$$

Let us calculate some definite integrals used in deriving eqns (4.5) and (5.12). Let us start from the following definite integral, which can be found in Lebedev and Silverman (1972) eqn (8.12.8) or it can be obtained from (A.1) and the orthogonality condition (3.4):

$$
\int_{0}^{\infty} \frac{\cosh \tau(\pi-|\beta|)}{\cosh \tau \pi} P_{-1 / 2+i \tau}(\cosh \alpha) d \tau=\frac{1}{\sqrt{2} \sqrt{\cosh \alpha-\cos \beta}}
$$

Recalling that

$$
\frac{d}{d \alpha} P_{-1 / 2+i \tau}(\cosh \alpha)=P_{-1 / 2+i \tau}^{1}(\cosh \alpha),
$$

and taking the derivative with respect to $\alpha$ of eqn (A.6) one gets 


$$
\int_{0}^{\infty} \frac{\cosh \tau(\pi-|\beta|)}{\cosh \tau \pi} P_{-1 / 2+i \tau}^{1}(\cosh \alpha) d \tau=-\frac{\sinh \alpha}{2 \sqrt{2}(\cosh \alpha-\cos \beta)^{3 / 2}} .
$$

Using the orthogonality relation (4.4), from (A.8) one gets

$$
\int_{0}^{\infty} \frac{P_{-1 / 2+i \tau}^{1}(\cosh \alpha)}{(\cosh \alpha-\cos \beta)^{3 / 2}} \sinh ^{2} \alpha d \alpha=-\frac{1+4 \tau^{2}}{\sqrt{2} \tau} \frac{\cosh \tau(\pi-|\beta|)}{\sinh \tau \pi} .
$$

Finally, by taking the derivative with respect to $\beta$ of eqn (A.9) one obtains

$$
\int_{0}^{\infty} \frac{P_{-1 / 2+i \tau}^{1}(\cosh \alpha)}{(\cosh \alpha-\cos \beta)^{5 / 2}} \sinh ^{2} \alpha d \alpha=-\frac{\sqrt{2}}{3} \frac{1+4 \tau^{2}}{\sin |\beta|} \frac{\sinh \tau(\pi-|\beta|)}{\sinh \tau \pi},
$$

which is also given in Miloh (1981), eqn (A.4).

From the definition of the incomplete Beta function (Gradshteyn and Ryzhik, 2007) one has

$$
\int_{c}^{\infty} \frac{y^{i t-1}}{y-\cos \beta} d y=(\cos \beta)^{i t-1} B\left(\frac{\cos \beta}{c}, 1-i t, 0\right) .
$$

\section{Appendix B. Evaluation of the subkernel $U(\tau, \eta)$}

Let us split the subkernel (3.6) for the problem of longitudinal heat flux into the sum

$$
U(\tau, \eta)=\int_{1}^{c} \frac{P_{-1 / 2+i \tau}(s) P_{-1 / 2+i \eta}(s)}{s-\cos \beta_{0}} d s+\int_{c}^{\infty} \frac{P_{-1 / 2+i \tau}(s) P_{-1 / 2+i \eta}(s)}{s-\cos \beta_{0}} d s,
$$

where $c>1$ is a constant. The first integral in (B.1) can be evaluated using a standard numerical integration scheme, such as the Gauss-Legendre quadrature for a finite interval, whereas the second integral can be approximated analytically for large $c$. According to Gradshteyn and Ryzhik (2007), the Legendre function $P_{-1 / 2+i \tau}(s)$ displays indeed the following asymptotic behavior as $s \rightarrow \infty$

$$
P_{-1 / 2+i \tau}(s)=\frac{1}{\pi} \sqrt{\frac{2}{s}} \operatorname{Re}\left[B\left(\frac{1}{2}, i \tau\right)(2 s)^{i \tau}\right]\left(1+O\left(s^{-2}\right)\right),
$$

where $B(p, q)$ is the Beta function. Therefore, for large $s$, the product of two Legendre functions can be approximated by

$$
\begin{aligned}
P_{-1 / 2+i \tau}(s) P_{-1 / 2+i \eta}(s)=\frac{1}{\pi^{2} s} & \operatorname{Re}\left[B\left(\frac{1}{2}, i \tau\right) B\left(\frac{1}{2}, i \eta\right)(2 s)^{i(\tau+\eta)}+\right. \\
& \left.+B\left(\frac{1}{2}, i \tau\right) B\left(\frac{1}{2},-i \eta\right)(2 s)^{i(\tau-\eta)}\right]\left(1+O\left(s^{-2}\right)\right)
\end{aligned}
$$


and thus, using the result (A.11) for $c>1$, the latter integral in (B.1) can be approximated by

$$
\begin{aligned}
& \int_{c}^{\infty} \frac{P_{-1 / 2+i \tau}(s) P_{-1 / 2+i \eta}(s)}{s-\cos \beta_{0}} d s=\frac{1}{\pi^{2} \cos \beta_{0}} \times \\
& \operatorname{Re}\left[B\left(\frac{1}{2}, i \tau\right) B\left(\frac{1}{2}, i \eta\right) B\left(\frac{\cos \beta_{0}}{c}, 1-i(\tau+\eta), 0\right)\left(2 \cos \beta_{0}\right)^{i(\tau+\eta)}+\right. \\
& \left.B\left(\frac{1}{2}, i \tau\right) B\left(\frac{1}{2},-i \eta\right) B\left(\frac{\cos \beta_{0}}{c}, 1-i(\tau-\eta), 0\right)\left(2 \cos \beta_{0}\right)^{i(\tau-\eta)}\right]\left(1+O\left(c^{-2}\right)\right),
\end{aligned}
$$

where $B(x, p, q)$ is the incomplete Beta function (Gradshteyn and Ryzhik, 2007).

Let us split the subkernel (4.6) for the problem of longitudinal heat flux into the sum

$$
U(\tau, \eta)=\int_{1}^{c} \frac{P_{-1 / 2+i \tau}^{1}(s) P_{-1 / 2+i \eta}^{1}(s)}{s-\cos \beta_{0}} d s+\int_{c}^{\infty} \frac{P_{-1 / 2+i \tau}^{1}(s) P_{-1 / 2+i \eta}^{1}(s)}{s-\cos \beta_{0}} d s,
$$

where $c>1$ is a constant. Similarly to (B.1), the first integral in (B.5) can be evaluated using a standard numerical integration scheme, whereas the second integral can be approximated analytically for large $c$. According to Gradshteyn and Ryzhik (2007), the Legendre function $P_{-1 / 2+i \tau}^{1}(s)$ displays the following asymptotic behavior as $s \rightarrow \infty$

$$
P_{-1 / 2+i \tau}^{1}(s)=-\frac{1}{\pi \sqrt{2 s}} \operatorname{Re}\left[B\left(-\frac{1}{2}, i \tau\right)(2 s)^{i \tau}\right]\left(1+O\left(s^{-2}\right)\right) .
$$

Therefore, for large $s$ the product of two Legendre functions of the first order can be approximated by

$$
\begin{aligned}
P_{-1 / 2+i \tau}^{1}(s) P_{-1 / 2+i \eta}^{1}(s)=\frac{1}{4 \pi^{2} s} & \operatorname{Re}\left[B\left(-\frac{1}{2}, i \tau\right) B\left(-\frac{1}{2}, i \eta\right)(2 s)^{i(\tau+\eta)}+\right. \\
& +B\left(-\frac{1}{2}, i \tau\right) B\left(-\frac{1}{2},-i \eta\right)(2 s)^{i(\tau-\eta)]\left(1+O\left(s^{-2}\right)\right) .}
\end{aligned}
$$

Using (A.11) for $c>1$, the second integral in (B.5) can be calculated as

$$
\begin{aligned}
& \int_{c}^{\infty} \frac{P_{-1 / 2+i \tau}^{1}(s) P_{-1 / 2+i \eta}^{1}(s)}{s-\cos \beta_{0}} d s=\frac{1}{4 \pi^{2} \cos \beta_{0}} \times \\
& \operatorname{Re}\left[B\left(-\frac{1}{2}, i \tau\right) B\left(-\frac{1}{2}, i \eta\right) B\left(\frac{\cos \beta_{0}}{c}, 1-i(\tau+\eta), 0\right)\left(2 \cos \beta_{0}\right)^{i(\tau+\eta)}+\right. \\
& B\left(-\frac{1}{2}, i \tau\right) B\left(-\frac{1}{2},-i \eta\right) B\left(\frac{\cos \beta_{0}}{c}, 1-i(\tau-\eta), 0\right)\left(2 \cos \beta_{0}\right)^{i(\tau-\eta)]\left(1+O\left(c^{-2}\right)\right) .}
\end{aligned}
$$




\section{FIGURES}
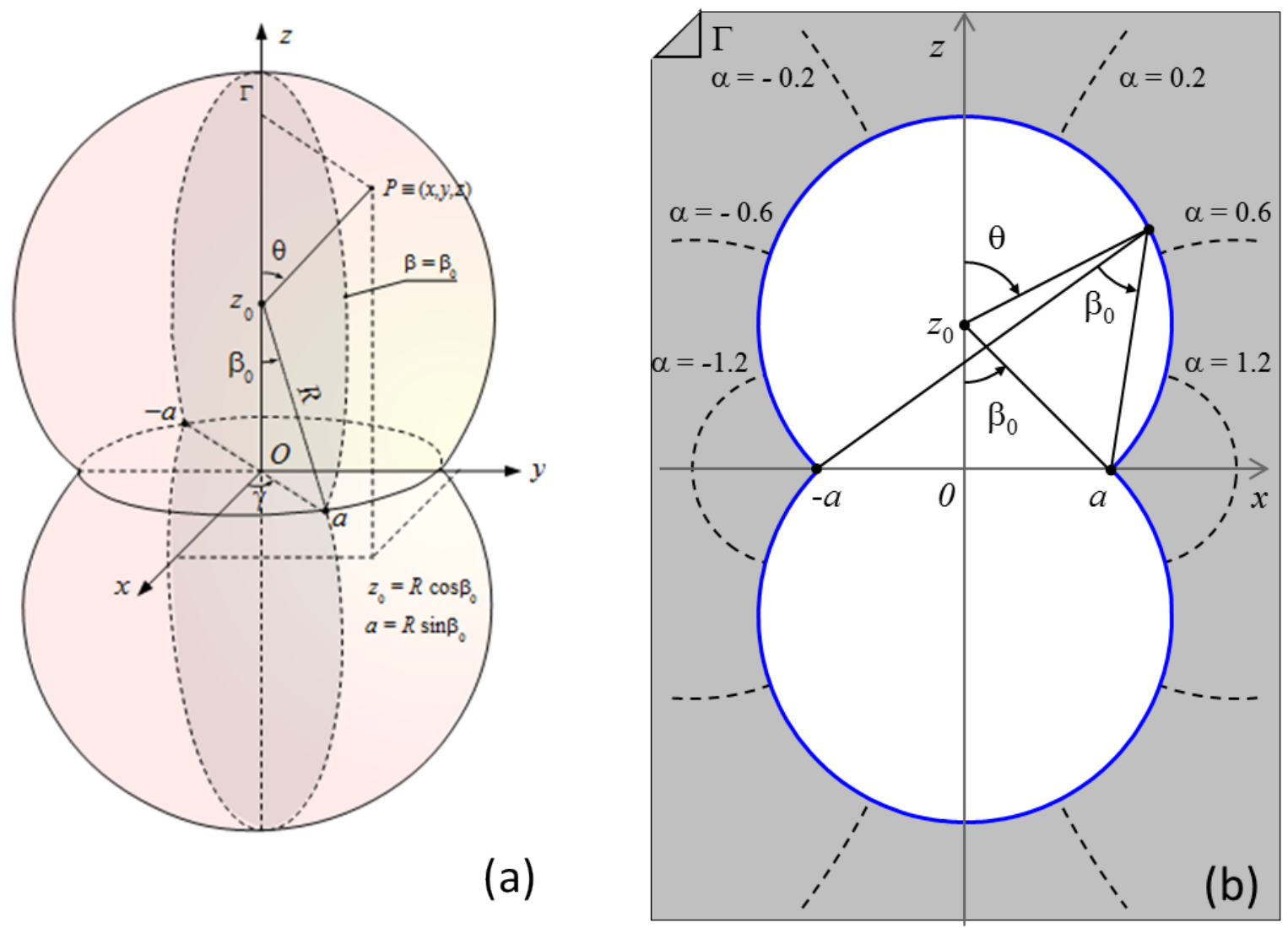

Figure 1. Double-sphere cavity defined by the toroidal coordinate $\beta=\beta_{0}$. (a) Three-dimensional view; (b) projection on xz plane. 


$$
\beta_{0}=0
$$$$
\beta_{0}=\pi / 4
$$$$
\beta_{0}=\pi / 2
$$$$
\beta_{0}=3 \pi / 4
$$
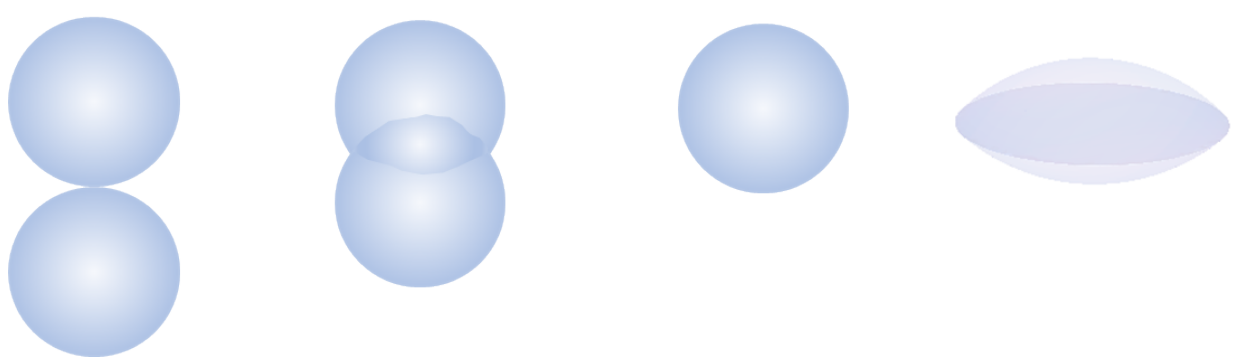

Figure 2. Variation of the shape of two coalesced spherical pores: from point contact to partial or complete coalescence, for $0 \leq \beta_{0} \leq \pi / 2$, and then from a sphere (complete coalescence) to a lentil and further to a penny-shaped crack, for $\pi / 2 \leq \beta_{0} \leq \pi$. 
a)

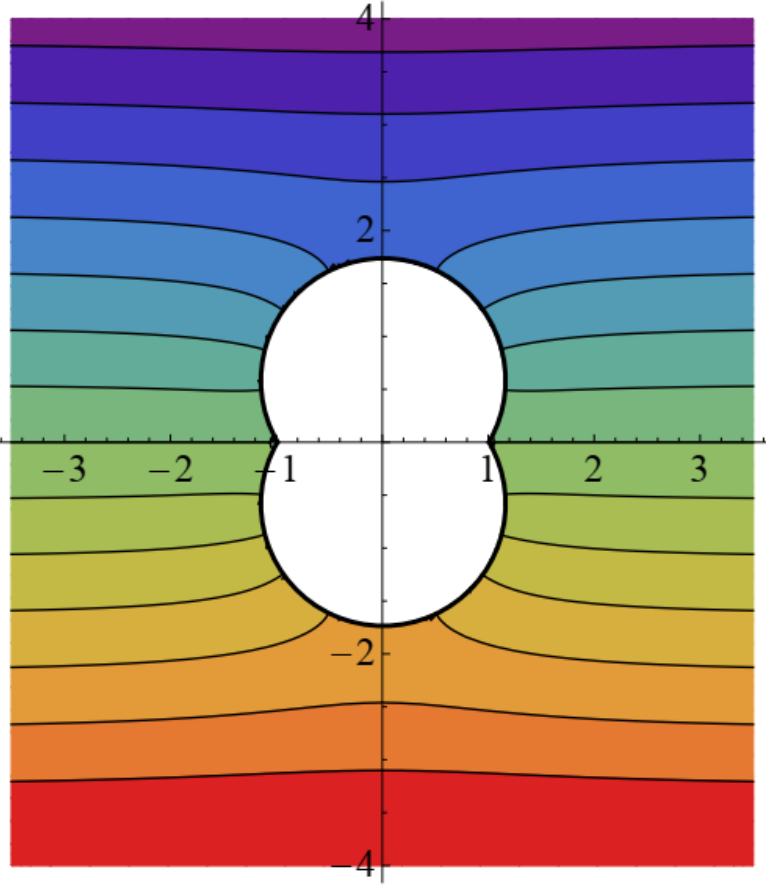

c)

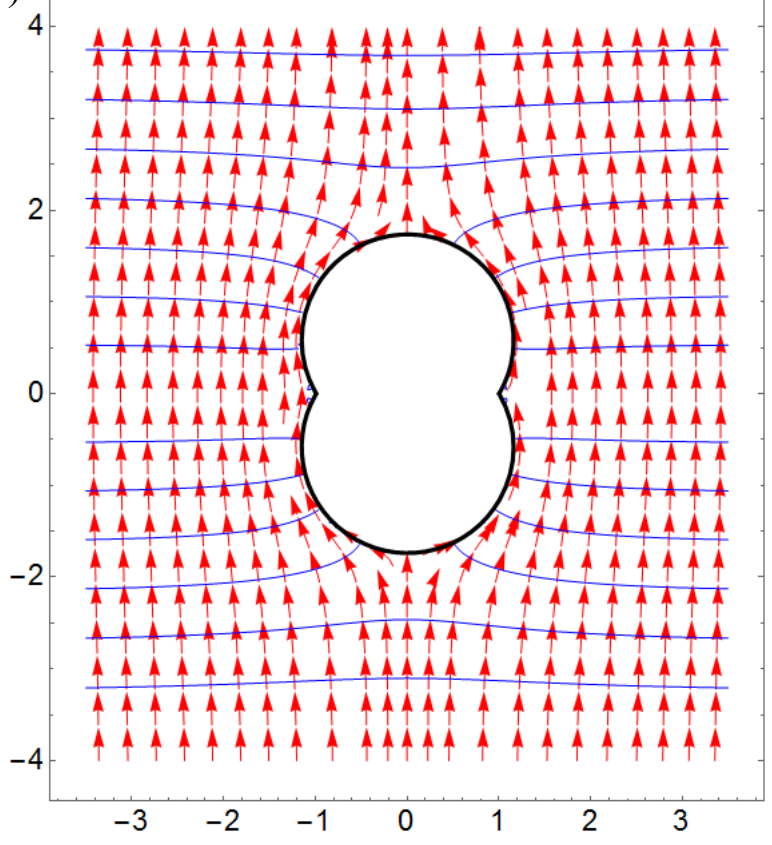

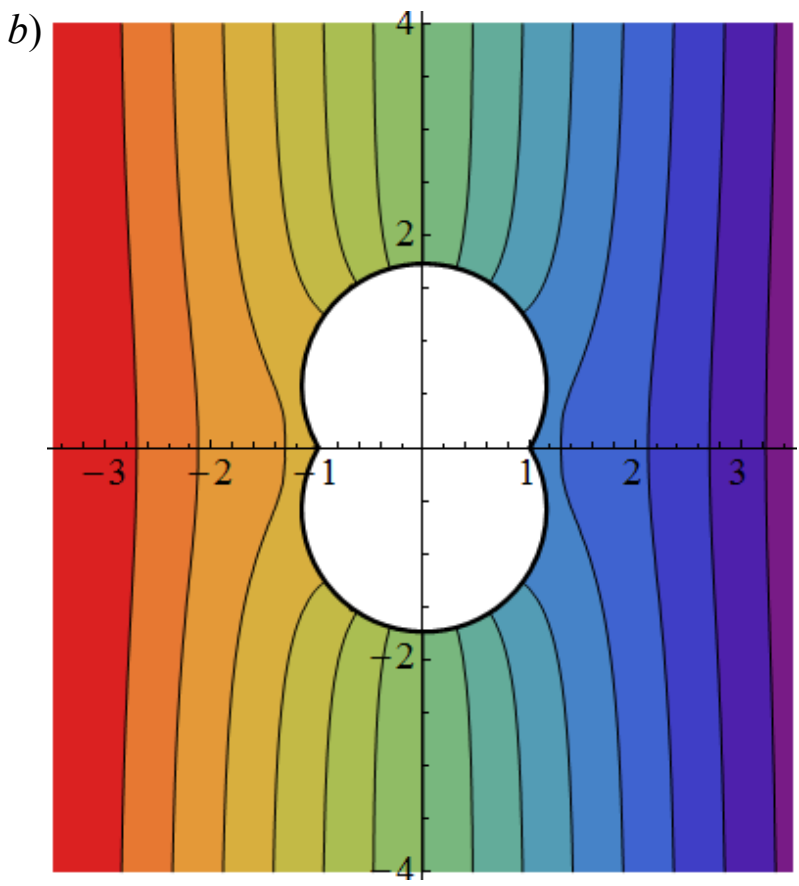

d)

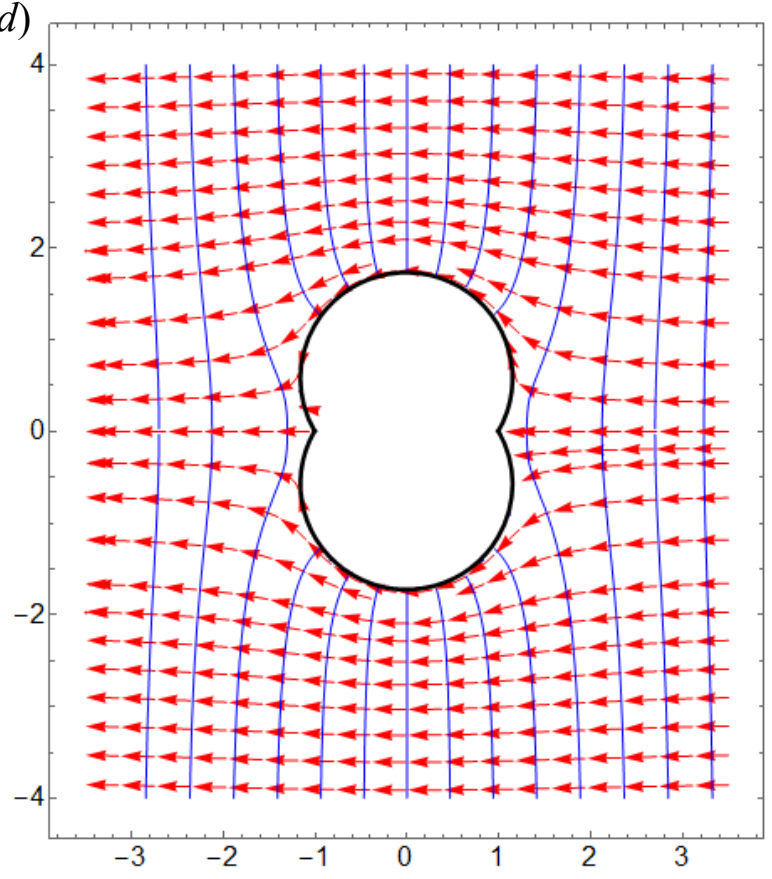

Figure 3. Temperature and heat flow fields for $\beta_{0}=\pi / 3$, for the remote heat flux $\boldsymbol{q}_{0}$ along the axial $(a$, $c)$ and transversal $(b, d)$ directions. 

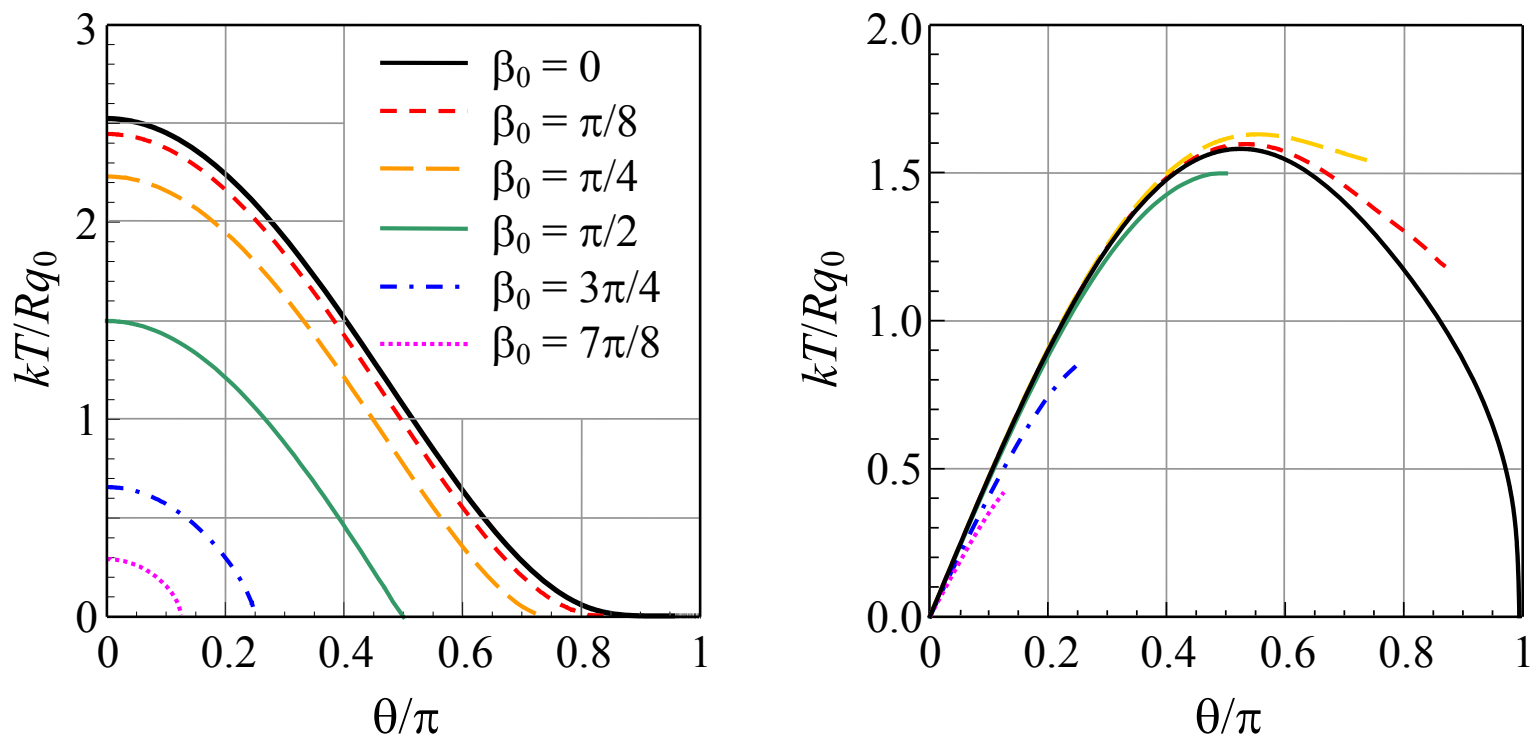

Figure 4. Temperature field on the surface of cavity defined by the toroidal coordinate $\beta=\beta_{0}$, for various value of $\beta_{0}$, for heat flux along the axial ( $a$ ) and transversal (b) directions. 

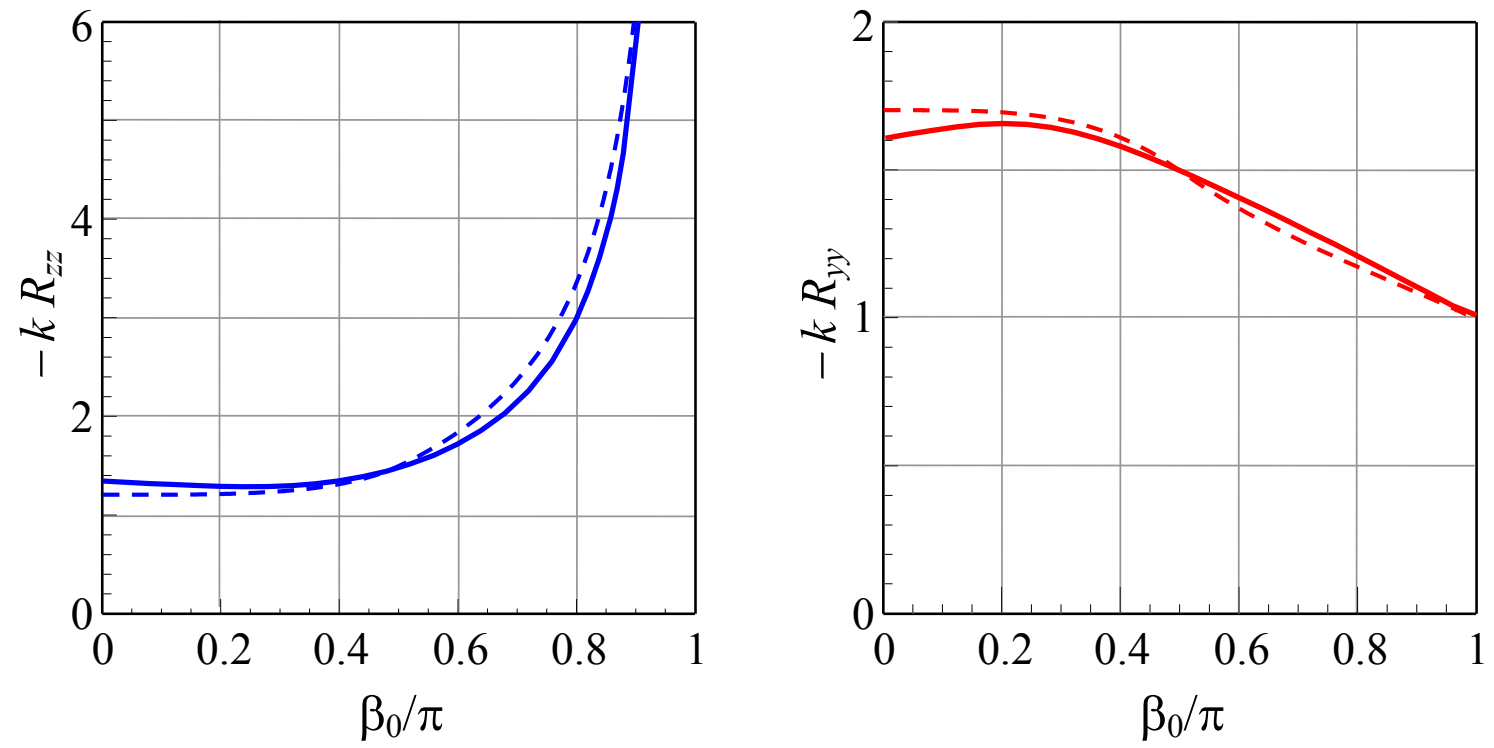

Figure 5. Normalized variation of the resistivity contribution tensor components with $\beta_{0}$. 


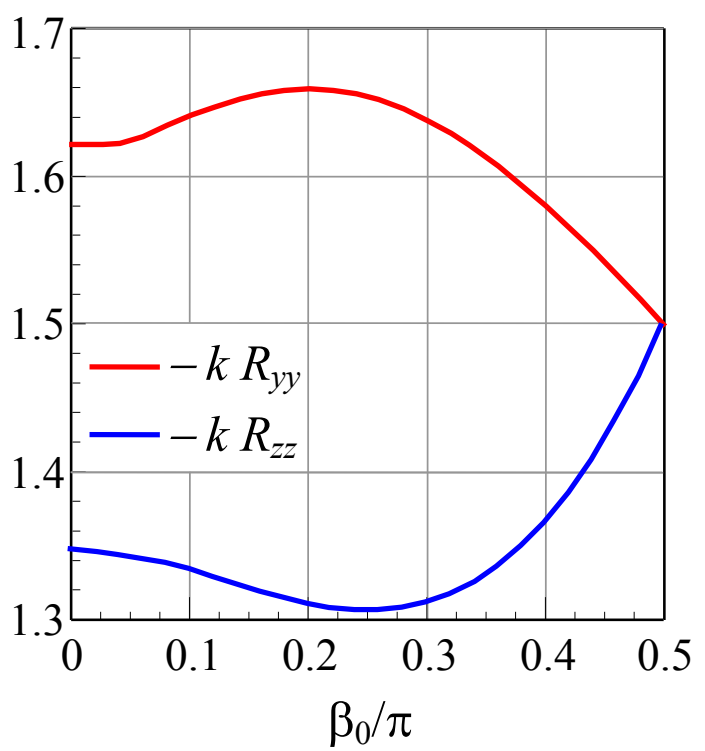

Figure 6. Zoom of Fig. 5 displaying the minimum of $R_{z z}$ and the maximum of $R_{y y}$. 
1) $\beta \leq \pi / 2$

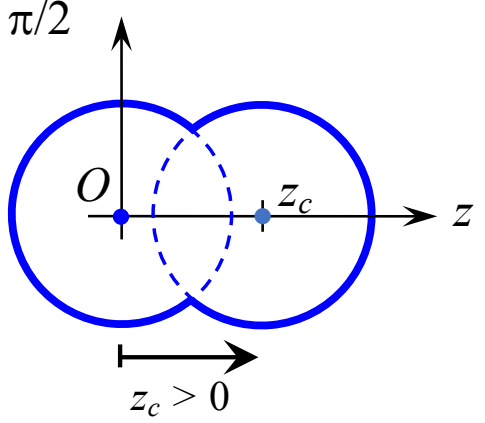

2) $\beta>\pi / 2$

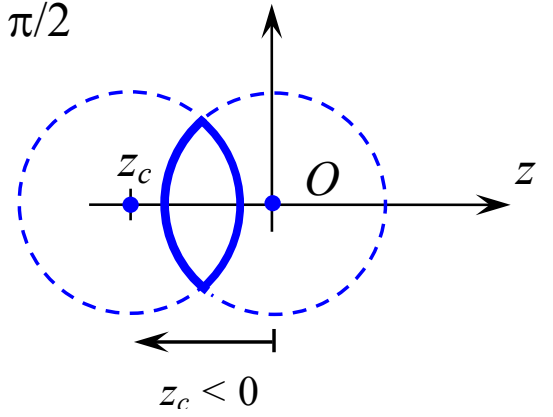

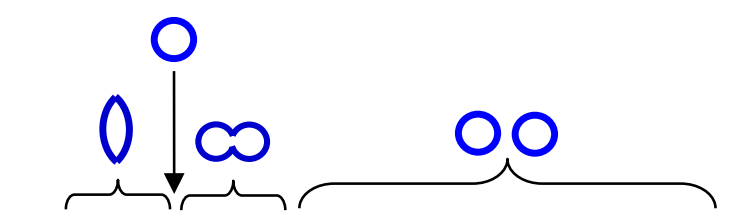

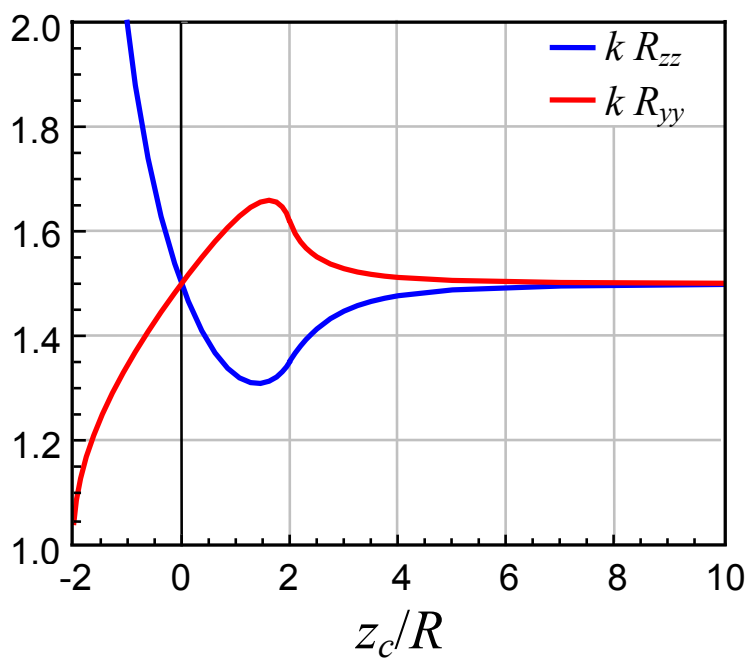

Figure 7. Variation of the components of the resistivity contribution tensor of two overlapped spheres as distance between their centers varies. Origin of the coordinate system is located at the centre of the fixed pore. The second pore moves from right to left. When the distance between pore centres $z_{\mathrm{c}}=2 z_{0}$ is much larger than $2 R$, the pores do not see each other and their joint resistivity contribution tensor coincides with that for a single sphere. When distance decreases, the magnitudes of the resistivity contribution tensor components is growing up reaching their maxima after the spheres coalesced. Then, a further pores coalescence process decreases the absolute values of the components down to that for a single sphere, recovered for $z_{\mathrm{c}}=0$. After that, the centre of the moving sphere is located to the left of the fixed sphere ("negative distance" $z_{\mathrm{c}}<0$ ) that corresponds to collapsing of a single pore. In the limiting case of $z_{\mathrm{c}}$ approaching -2 , we recover the values of the resistivity contribution tensor corresponding to a penny shaped crack. 


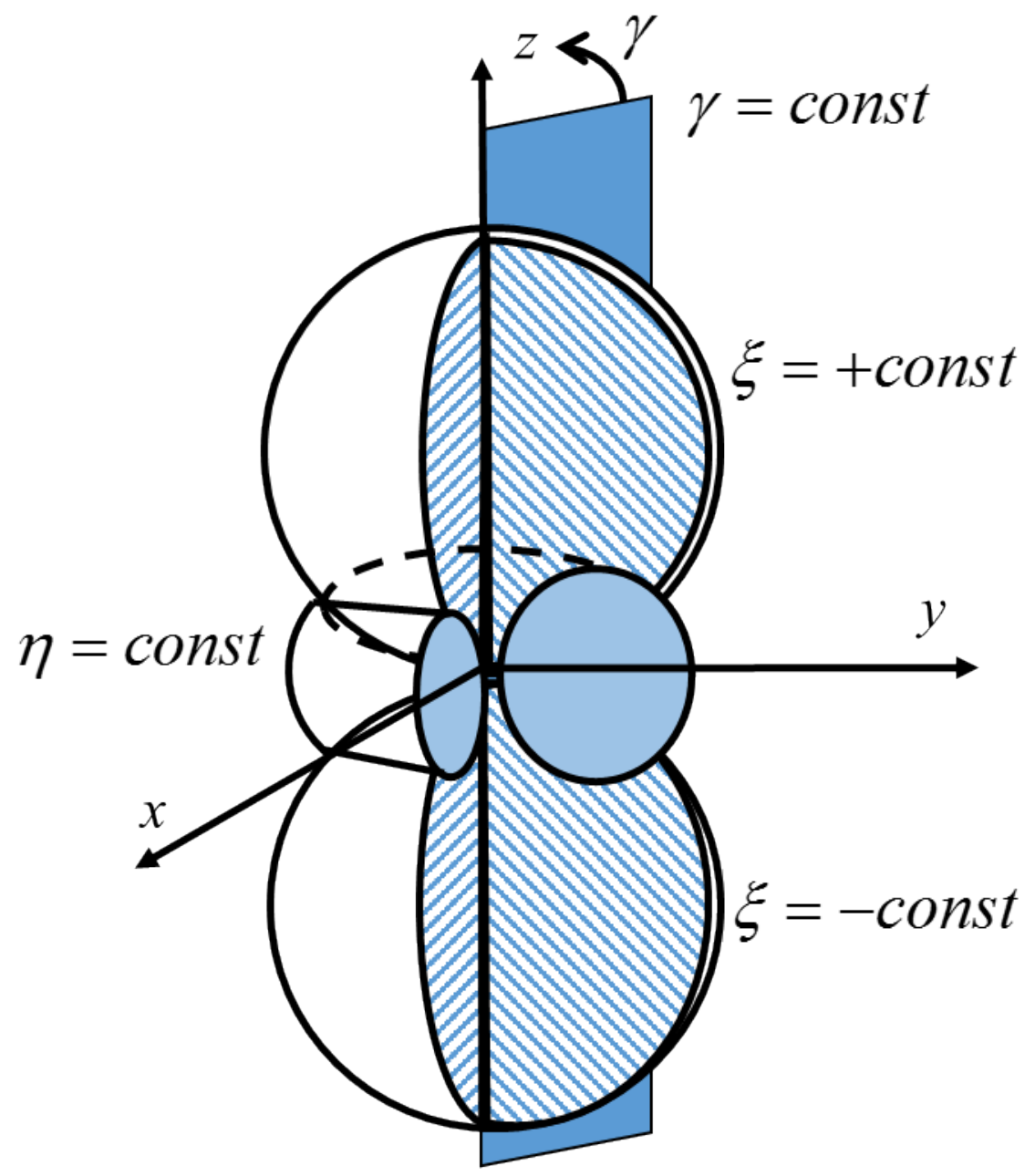

Figure 8. Tangent sphere coordinate system $(\xi, \eta, \gamma)$ 

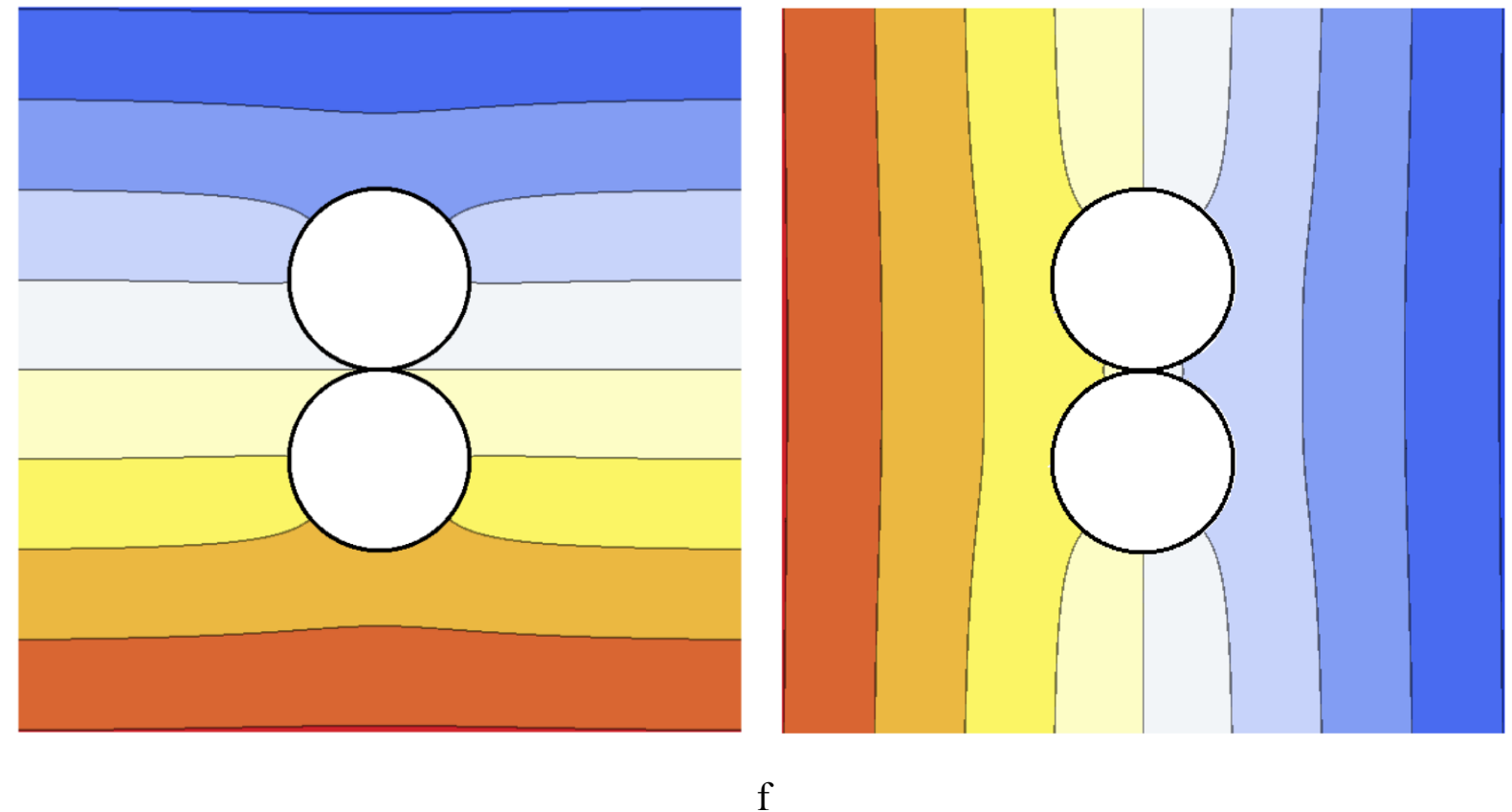

Figure 9. Temperature fields for two touching spheres, for heat flux along the axial $(a)$ and transversal (b) directions. 


\section{Declaration of interests}

૫ The authors declare that they have no known competing financial interests or personal relationships that could have appeared to influence the work reported in this paper.

$\square$ The authors declare the following financial interests/personal relationships which may be considered as potential competing interests:

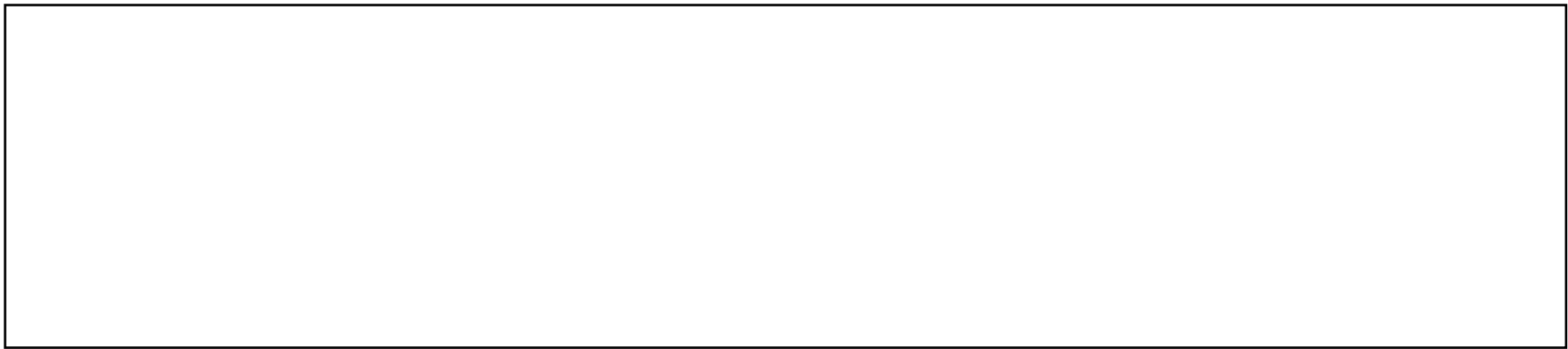




\section{Luca Lanzoni}

Formal analysis; Visualization

\section{Enrico Radi}

Methodology; Data curation; Writing - original draft

Igor Sevostianov

Conceptualization; Writing -review \& editing; Visualization 